\title{
Enable the Landing of Internet of Things: A Holistic Approach
}

\section{Yu Liu}





\title{
Enable the landing of Internet of Things: a holistic approach
}

\author{
Yu Liu
}

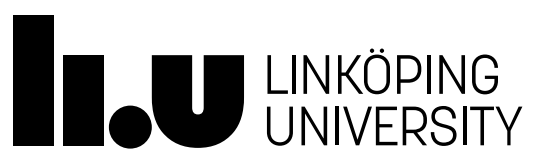

Linköping University

Department of Science and Technology

Division of Physics, Electronics and Mathematics

SE-601 74 Norrköping, Sweden

Norrköping 2021 
$(\mathrm{cos})$ EY-No NonCommercial 4.0 International License.

https://creativecommons.org/licenses/by-nc/4.0/

Edition 1:1

Copyright @ $\odot$ Yu Liu, 2021

ISBN 978-91-7929-670-4

ISSN 0345-7524

Published articles have been reprinted with permission from the respective copyright holder.

Typeset using $\mathrm{X}_{\mathrm{H}} \mathrm{T}_{\mathrm{E}} \mathrm{X}$

Printed by LiU-Tryck, Linköping 2021 


\section{POPULÄRVETENSKAPLIG SAMMANFATTNING}

Visionen med Sakernas internet (IoT) är en värld där fysiska apparater är uppkopplade i en sådan grad att dess digitaliseringslösningar ger verklig samhällsnytta. Framsteg inom informations och kommunikationsteknologi har lett till utveckling av IoT-applikationer för olika ändamål. Dessa applikationer förväntas att få en betydande roll för samhället i stort såväl som i industriella sammanhang. Framväxten av molntjänster med kraftfulla lagrings, beräknings, analys och visualiseringsmöjligheter har accelererat användningen av IoT-teknologi. Trots den snabba utvecklingen så finns det flera utmaningar kvar, som exempelvis kompatibilitet mellan olika protokoll, delsystem och olika underliggande tjänster. Ett annat exempel är heterogenitet vad gäller datainsamling och kommunikation, vilket det ännu inte finns någon lösning för hos molntjänsteleverantörerna. I den här avhandlingen presenteras ett IoT-ramverk baserat på publika molntjänster som adresserar dessa utmaningar. Ramverket inkluderar stöd för WiFi, Thread och LoRaWAN nätverk för att möjliggöra ett brett utbud av IoT-applikationer. En taxonomi för säkerhetsbedömning av olika delar i berörda IoTnätverk ingår också. Som en följd av att allt mer av beräkningarna i molnet decentraliserats ut i ändnoderna så har IoT-ramverket designats med hänsyn till det. Inkluderat är också en omfattande prestandaanalys av IoT-ramverkets stack för beräkningar i ändnoderna, i vilken kommunikation, beräkning, samt maskinlärning har utvärderats. En plattform med demonstratörer baserad på IoT-ramverket har designats och realiserats för växtväggsindustrin. Plattformen har även använts för att demonstrera hur maskininlärning kan tillämpas för att ge växtväggar intelligens att upptäcka anomalier. Baserat på detta forskningsarbete och erfarenheter från pilotinstallationer, så har en digitaliseringsmetodik utarbetats för att guida små och medelstora företag i den digitala transformation som IoT-teknologin medför. På detta sätt har en sann landning av IoT-tekniken i samhället demonstrerats. 


\begin{abstract}
Internet of Things (IoT) envisions a world where physical assets are fully connected with the Internet infrastructure to provide digital services. With the advancement of information and communication technologies, IoT applications have experienced a growth in many industries and are anticipated to reshape the landscape of social life and industry production. The emergence of cloud computing has accelerated the widespread employment of IoT technologies, benefiting from superb computation, storage, analytics and visualization capabilities. However, the landing of IoT still encounters several open challenges, i.e., interoperability and compatibility between link layer protocols, subsystems, and back-end services. Moreover, a uniform scheme for device management and the heterogeneity of data have not been tackled by cloud suppliers. In this dissertation, a data-centric IoT framework based on public cloud is presented to address these challenges. It features $\mathrm{WiFi}$, Thread, and LoRaWAN networks to provide support for personal, local and wide area networks so as to enable wide coverage of IoT applications. A security analysis taxonomy is proposed to perform security assessment of IoT field networks and enhance security considerations. In light of the recent industrial tendency that cloud computing is evolving towards edge-cloud computing, further reinforcement of the IoT framework is proposed with the novel edge-cloud computing paradigm. A comprehensive performance evaluation of the edge-cloud computing stack is conducted, while the communication, computing and intelligence capabilities are thoroughly studied for future cloud and edge computing enabled IoT applications. Furthermore, the cloud and edge computing enabled IoT landing with a digitalization practice is showcased in the vertical plant wall industry. A remote monitoring and management system for indoor climate control has been developed based on the IoT framework. As a further step, it is also demonstrated how machine learning can be leveraged to achieve artificial intelligence in IoT with a case study, i.e., anomaly detection for indoor climate. Based on the expertise we accumulated from the industry digitalization practice, a reference framework that intends to guide small and medium sized enterprises to perform IoT enabled digital transformation is proposed. In this way, a true landing of the IoT technology in the society has been demonstrated.
\end{abstract}




\section{Acknowledgments}

Four years Ph.D. study is a unique journey to me, and now it is approaching an end. Like many Ph.D. researchers, during the four years, I experienced confusions and upsetting time when I was struggling hard to find the significance of the work I was doing, and also derived great enjoyment from the research results that were endorsed by colleagues in the academia and the industry. The lessons I learned from my Ph.D. career not only strengthen my research competence but also make me a mature thinker, which I will benefit for the whole life.

In the last four years, I received many help, support, and encouragement from my colleagues and friends. I would like to show my heartful thanks to them. Especially, I would like to thank my supervisors, Professor Shaofang Gong, Associate Professor Magnus Karlsson, and Doctor Zhibo Pang. Shaofang is a great supervisor and a warm guider. He offered me this opportunity to start my research career, provided me with a comfortable and flexible work environment, and cared me in both work and life. His encouragement gave me so much confidence. Magnus offered me great support and valuable input in research and in life, trained me with practical skills and impressed me with his serious attitude in doing everything. Zhibo always shared insightful views with me, gave me guidance from a mature industrial scientist and practitioner's perspective. Without their support, I am a lesser man.

I would like to thank my colleagues who are within or stayed in the Communication Electronics research group, FEM division and ITN department, Adriana Serban, Gustav Knutsson, Xin Xu, Qin-Zhong Ye, Robert Malmqvist, Zhongjun Ni, Zhuangwei Liu, and Rasmus Ringdahl, for their valuable input and support to my study, research, and life. Thanks to Associate Professor Vangelis Angelakis who showed me another methodology to conduct research. Special thanks to Professor Michael Hörnquist who genuinely helped me a lot to stay and to develop.

Moreover, I would like to show my appreciation to my colleagues and partners within the projects I was involved, Ola Weister, Kahin Akram Has- 
san, Professor Anita Lloyd Spetz, Docent Donatella Puglisi, Tomas Uusitalo, Åke Johansson, and Patrik Arven, for their trust and support.

I sincerely thank the company of my dear colleagues and friends from the Chinese students and scholars association and in my life. I do enjoy the friendship and fun we had.

Vinnova, Swedish Environmental Protection Agency, and Norrköping Fund for Research and Development in Sweden are acknowledged for financial support of the Ph.D. study.

Last but not least, I want to express my deepest gratitude to my family, my wife Xinchen Wang, my parents and my parents in law. Without your firm support behind me, I could not have gone so far.

Yu Liu,

Norrköping, March 2021 


\section{List of Publications}

\section{Papers included in this thesis:}

Paper I: Y. Liu, K. Akram Hassan, M. Karlsson, Z. Pang and S. Gong, "A Data-Centric Internet of Things Framework Based on Azure Cloud," in IEEE Access, vol. 7, pp. 53839-53858, 2019.

Paper II: Y. Liu, Z. Pang, G. Dán, D. Lan and S. Gong, "A Taxonomy for the Security Assessment of IP-Based Building Automation Systems: The Case of Thread," in IEEE Transactions on Industrial Informatics, vol. 14, no. 9, pp. 4113-4123, Sept. 2018.

Paper III: Y. Liu, D. Lan, Z. Pang, M. Karlsson and S. Gong, "Performance evaluation of containerization in edge-cloud computing stacks for industrial applications: a client perspective," in IEEE Open Journal of the Industrial Electronics Society, vol. 2, pp. 153-168, 2021.

Paper IV: Y. Liu, K. Akram Hassan, M. Karlsson, O. Weister and S. Gong, "Active Plant Wall for Green Indoor Climate Based on Cloud and Internet of Things," in IEEE Access, vol. 6, pp. 33631-33644, 2018.

Paper V: Y. Liu, M. Karlsson, Z. Pang and S. Gong, "Anomaly detection based on machine learning in IoT-based vertical plant wall for indoor climate control," in Building and Environment, vol. 183, 2020.

Paper VI: Y. Liu, Z. Ni, M. Karlsson, Z. Pang and S. Gong, "Methodology for digital transformation with Internet of things and cloud computing: a practical guideline for innovation to small and medium-sized enterprises", Manuscript, 2021.

\section{Papers not included in this thesis:}

Z. Ni, P. Eriksson, Y. Liu, M. Karlsson and S. Gong, "Improving energy efficiency while preserving historic buildings with digital twins and artificial intelligence" in the 2021 Sustainable Built Heritage Conference, Bolzano, Italy, 
2021.

D. Lan, Y. Liu, A. Taherkordi, F. Eliassen and L. Liu, "A Federated Fog-Cloud Framework for Data Processing and Orchestration: a Case Study of Smart Green-wall in Smart Cities" in the 2021 ACM/SIGAPP Symposium on Applied Computing, Gwangju, Korea, 2021.

K. Akram Hassan,Y. Liu, L. Besançon, J. Johansson, and N. Rönnberg, "A Study on Visual Representations for Active PlantWall Data Analysis," in Data 4.2, 2019.

D. Lan, Z. Pang, C. Fischione, Y. Liu, A. Taherkordi and F. Eliassen, "Latency Analysis of Wireless Networks for Proximity Services in Smart Home and Building Automation: The Case of Thread," in IEEE Access, vol. 7, pp. 4856-4867, 2019.

E. Azoidou, Z. Pang, Y. Liu, D. Lan, G. Bag and S. Gong, "Battery Lifetime Modeling and Validation of Wireless Building Automation Devices in Thread," in IEEE Transactions on Industrial Informatics, vol. 14, no. 7, pp. 2869-2880, July 2018. 


\section{List of Abbreviations}

$\begin{array}{ll}\text { IoT } & \text { Internet of Things } \\ \text { M2M } & \text { Machine to Machine } \\ \text { AIoT } & \text { Artificial Intelligence and Internet of Things } \\ \text { IIoT } & \text { Industrial Internet of Things } \\ \text { HSC } & \text { Hyper-scale Cloud } \\ \text { PaaS } & \text { Platform as a Service } \\ \text { SaaS } & \text { Software as a Service } \\ \text { SME } & \text { Small and Medium-sized Enterprise } \\ \text { LAN } & \text { Local Area Network } \\ \text { PAN } & \text { Personal Area Network } \\ \text { WAN } & \text { Wide Area Network } \\ \text { HTTPS } & \text { Hypertext Transfer Protocol Secure } \\ \text { AMQP } & \text { Advanced Message Queuing Protocol } \\ \text { MQTT } & \text { Message Queuing Telemetry Transport } \\ \text { MQTT-SN } & \text { MQTT for Sensor Network } \\ \text { IP } & \text { Internet Protocol } \\ \text { JSON } & \text { JavaScript Object Notation } \\ \text { HMI } & \text { Human Machine Interface } \\ \text { CoAP } & \text { Constrained Application Protocol } \\ \text { D2C } & \text { Device to Cloud } \\ \text { Cloud Device }\end{array}$


BAN Building Automation Network

BAS Building Automation System

DoS Denial of Service

OS Operating System

TLS Transport Layer Security

DTLS Datagram Transport Layer Security

WPA WiFi Protected Access

KRACK Key Reinstallation Attack

VPN Virtual Private Network

AES Advanced Encryption Standard

RTT Round Trip Time

SoC System on Chip

VM Virtual Machine

LSTM-ED Long Short-term Memory Encoder-Decoder

PM Particulate Matter

VOC Volatile Organic Compound

VPS Vertical Plant System

ML Machine Learning

AI Artificial Intelligence

ANN Artificial Neural Network

AE Auto-encoder

RNN Recurrent Neural Network

MAE Mean Absolute Error

MSE Mean Squared Error

ROC Receiver Operating Characteristic

AUC Area Under RoC Curve

SWD Sliding Window 
OSWD Overlapped Sliding Window

TPR True Positive Rate

FPR False Positive Rate

ICT Information and Communication Technology

SDK Software Development Kit 



\section{Contents}

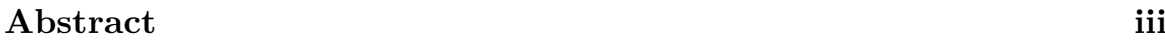

Acknowledgments vi

List of Publications viii

List of Abbreviations $\quad$ xi

Contents $\quad$ xiii

1 Introduction $\quad 1$

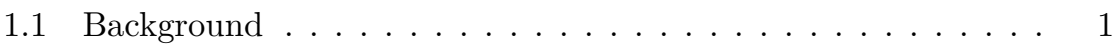

1.2 IoT segmentation . . . . . . . . . . . . . . . 2

1.3 Enabling IoT landing: from cloud to edge computing . . . . . . 3

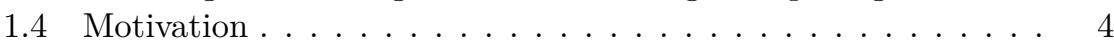

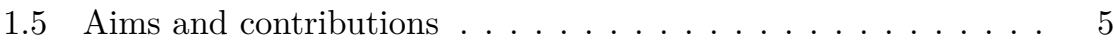

1.6 Thesis outline . . . . . . . . . . . . . . 6

2 Cloud-enabled IoT $\quad 7$

2.1 Framework overview . . . . . . . . . . . . . . . 7

2.2 Azure cloud infrastructure . . . . . . . . . . . . . 8

2.3 Local infrastructure . . . . . . . . . . . . . . . . . . . . . . . . . . . . . . . . . 10

2.4 Device model . . . . . . . . . . . . . . . . . . . 12

2.5 Data model ...................... 13

3 Security of the framework $\quad \mathbf{1 5}$

3.1 Security analysis taxonomy . . . . . . . . . . . . . 15

3.2 Security assessment of the framework . . . . . . . . . . . 17

4 From cloud to edge computing $\quad 19$

4.1 Edge-cloud computing stack . . . . . . . . . . . . . . . 19

4.2 Performance evaluation of edge-cloud architecture . . . . . . . 21

5 Case study: digitalization in the vertical plant wall industry 27 
5.1 Background ......................... 27

5.2 System overview . . . . . . . . . . . . . . . . . . . . . . . . . . . . . . . . . . . . 28

5.3 Implementation . . . . . . . . . . . . . . . . . . . . . . . . 29

5.4 Evolving to the edge-cloud architecture . . . . . . . . . 31

6 Intelligence in IoT $\mathbf{3 5}$

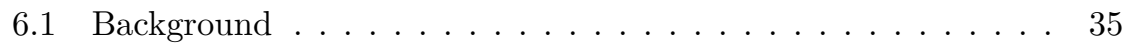

6.2 Anomaly detection for indoor climate control . . . . . . . . . 36

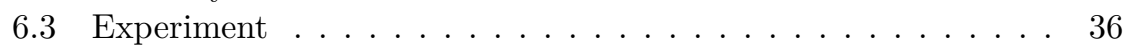

7 A methodology for digital transformation $\quad 41$

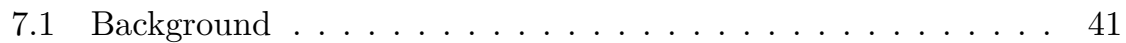

7.2 Digital transformation framework . . . . . . . . . . . . . . . . 42

7.3 Design principles . . . . . . . . . . . . . . . . 43

8 Summary of Publications $\quad 45$

9 Conclusion $\quad 49$

$\begin{array}{ll}\text { Bibliography } & 51\end{array}$

$\begin{array}{ll}\text { Paper I } & 57\end{array}$

$\begin{array}{lr}\text { Paper II } & 79\end{array}$

$\begin{array}{ll}\text { Paper III } & 93\end{array}$

$\begin{array}{ll}\text { Paper IV } & 113\end{array}$

$\begin{array}{lr}\text { Paper V } & 129\end{array}$

$\begin{array}{ll}\text { Paper VI } & 145\end{array}$ 


\section{Introduction}

\subsection{Background}

The term Internet of things (IoT) has been proposed to reflect a full connectivity between the physical world and the digital world through the Internet infrastructure. The IoT paradigm has been pervasively adopted in various research domains and applications, which enables new terms such as smart city, smart agriculture, smart factory and smart home to be created and reshape human daily life and industry production [1]. In short, the tremendous influence of IoT on the modern society has been clearly observed.

IoT originates from the traditional machine to machine (M2M) communication technology which can date back to the last century and constitutes the foundation of IoT. The concept of M2M emphasizes point to point communication between devices using customized communication channels. IoT envisions a broader blueprint where large-scale connectivity among a variety of things, i.e., devices, sensors, actuators and processing platforms, through distinct wired or wireless networks and communication protocols can be established. Within such a network, the interactions among devices are enhanced, and large volume of data can be collected and analyzed so as to develop intelligence - the crown of the big data era. The exploitation of IoT has been prevalent due to the continuously developed Internet infrastructure, network capabilities, reduced cost, and more and more open standards [2]. Considering the increased complexity in data communication and computation, in recent years, the maturation of cloud computing has truly accelerated the 


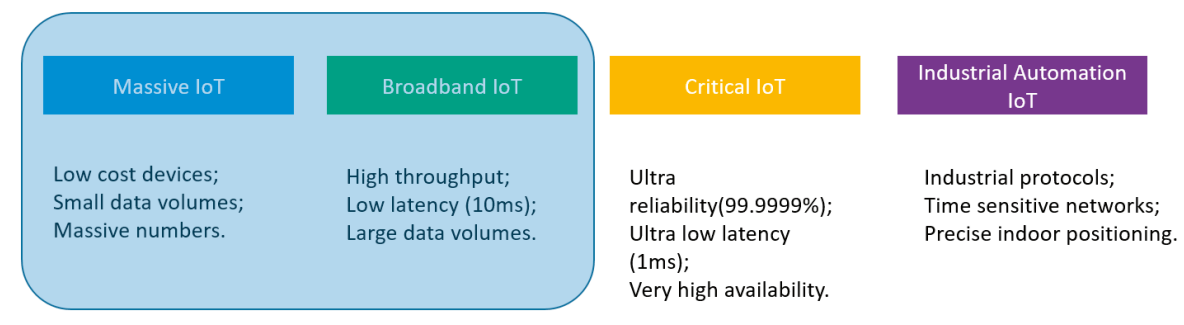

Figure 1.1: IoT applications are categorized into four segments according to different performance requirements [3].

landing of IoT in all applications of the society, benefiting from the centralized data acquisition, flexible and scalable storage, and powerful computation capabilities.

\subsection{IoT segmentation}

As shown in Figure 1.1, according to different performance requirements on latency, data volume, throughput, reliability and availability, IoT applications are segmented into four groups, namely Massive IoT, Broadband IoT, Critical IoT, and Industrial Automation IoT [4]. Massive IoT aims at low cost and low complexity devices that are with small data volume and low data rate but deployed in large coverage. Broadband IoT is proposed for high throughput and low latency applications with large volume of traffic. These two categories can support a majority of IoT scenarios such as monitoring, metering, logistics, and building automation in the smart city paradigm, due to the non-stringent performance requirements $[5,6]$. The rest two categories characterizing much stringent requirements are aiming for industry IoT (IIoT) applications.

Some quantitative requirements of IoT applications can be found in the literature. For instance, the latency requirement in industrial automation is much demanding, e.g., $<2 \mathrm{~ms}$ cycle time for motion control, $2-10 \mathrm{~ms}$ for factory automation, $50 \mathrm{~ms}$ for process monitoring and 10-100 ms for videooperated remote control [7] while latency is relatively tolerable in the massive IoT category, e.g., 40-500 ms for traffic management, $<1 \mathrm{~s}$ for audio and video transfer in smart grid [8], etc. The payload in industrial communication can range from a few bytes for process automation up to 250 bytes for machine control [7] and 1.5 kilobytes for smart grid [9]. Considering the number of concurrent nodes, typically $>20$ are deployed for high-mobility nodes and 10000 are required for low-mobility nodes [7]. 


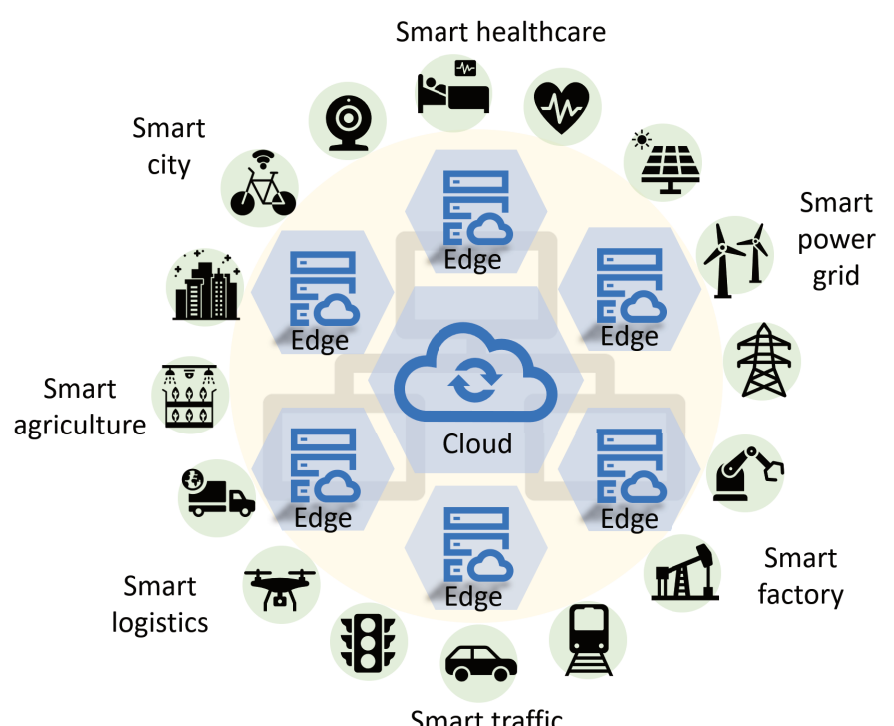

Smart traffic

Figure 1.2: An overview of the edge-cloud computing paradigm used in different scenarios, e.g, smart city, agriculture, logistics, traffics, factory, power grid, and healthcare. Edge devices are connected to the cloud platform via Ethernet while the communications between field and edge devices are through heterogeneous wired or wireless protocols [10].

\subsection{Enabling IoT landing: from cloud to edge computing}

The advancement of cloud computing has enabled widespread deployment of cloud-enabled IoT applications. With public cloud platform, services that are commonly employed in IoT solutions such as device management, data processing and storage, and analytics, can be flexibly subscribed and combined while the efforts and resources of IoT practitioners can be dedicated to realization of core functions that are more essential to business. In short, high reliability, scalability and low cost are the key advantages to drive the landing of IoT with cloud computing.

As a result of the technological revolution, a tendency has been observed that the edge-cloud computing paradigm is replacing the cloud computing paradigm, to resolve the challenges and facilitate IoT innovations to be adopted. As shown in Figure 1.2, with edge computing units deployed to the proximity of network edge, IoT applications can experience low latency and fast decision-making from the edge device while reducing the bandwidth towards the cloud platform so as to provide enhanced privacy and security. Additionally, artificial intelligence can be deployed to the edge device, which 
brings intelligence closer to the user scenarios and fosters novel machine learning methods. Catering to the heterogeneity of hardware and software in edge computing infrastructures, microservice and the container technology based virtualization become a promising approach in edge computing practice. Microservices splits an application into small modules with little dependency on each other while the container technology becomes an optimal carrier for microservices due to lightweight and low footprint. Therefore, container-based virtualization is a key enabler to the success of edge computing in the context of IoT, which has been a consensus of hyper-scale cloud (HSC) and network infrastructure suppliers [11].

\subsection{Motivation}

The cloud computing and edge computing paradigms are enabling the landing of IoT. This has been confirmed by the cloud computing industry that major public cloud suppliers, e.g., Amazon Web Service, Google Cloud Platform, and Microsoft Azure Cloud, have all released their infrastructures to cater to the needs of IoT applications, from cloud towards the edge device level.

However, there are still open questions and challenges hindering the industry from fully utilizing IoT in practice. To incorporate a variety of end devices and systems while providing digital services and extracting information from accumulated data, a uniform procedure of data collection, aggregation, transmission, storage, processing and visualization must be defined in a universal IoT framework. Existing infrastructures offered by the cloud industry can hardly realize such a goal due to these challenges:

- First, IoT includes a collection of public or proprietary standards and protocols, therefore, the interoperability and compatibility between different link layer protocols, sub-systems and back-end services can be challenging. This challenge is not within the business scope of cloud suppliers who merely focus on software as a service (SaaS) and platform as a service (PaaS) and cannot reach the communications among end devices due to the heterogeneity of hardware and protocols.

- Second, the diversity of IoT technologies and application scenarios makes it challenging to integrate device commissioning, communication, authorization, as well as identity registration and management into a uniform security scheme.

- Last, the heterogeneity of data format, data rate, and storage mechanism poses a challenge for the utilization of large volume of IoT data to harvest intelligence.

The emerging edge-cloud computing paradigm is now in the ascendant and its developing foreground is vast. Particularly, the container-based virtualiza- 
tion technology has been put on the roadmap by the industry to implement edge-cloud computing infrastructure. In this regard, the following questions are still unclear and worth a thorough investigation.

- Has the performance of the container-based edge-cloud computing stacks reached industry requirement?

- How much room of the performance of the state-of-the-art edge-cloud computing infrastructure shall be improved to reach the industrial anticipation?

Last but not the least, IoT is seen as one of the key technologies to drive digital transformation in industry. Restricted by limited resources and lack of expertise in IoT technologies, many traditional industries, especially small and medium sized enterprises (SMEs) have difficulty to perform an digital transformation.

- Therefore, a reference framework that can be followed as a guideline to accelerate digital transformation for SMEs is under demand.

\subsection{Aims and contributions}

This thesis aims to address the aforementioned challenges and those unclear questions. Specifically, this thesis contributes to the academic and industrial community in the following aspects.

1. Proposed a data-centric IoT framework exemplified with three most representative protocols to cover the local area network (LAN), personal area network (PAN) and wide area network (WAN) use cases.

2. Eased the interoperability, co-existence, device management and data consistency challenges in the IoT practice with public cloud infrastructure.

3. Proposed a security analysis taxonomy for wireless communication practices in IoT to enhance the security consideration.

4. Performed a comprehensive performance evaluation to investigate communication, computing, and intelligence capabilities on the state-of-theart edge-cloud computing infrastructures.

5. Profiled the performance limitation and highlighted the necessity of partitioning in edge-cloud computing.

6. Exemplified cloud and edge computing enabled IoT landing in a real industry application, and showcased how machine learning and artificial intelligence are leveraged in IoT practice. 
7. Proposed a reference framework to accelerate IoT and cloud computing enabled digital transformation to take place in SMEs.

\subsection{Thesis outline}

The remainder of the thesis is organized as follows. In Chapter 2, the proposed data-centric IoT framework based on public cloud platform is introduced. In Chapter 3, a security analysis taxonomy for wireless communication in IoT is presented and the security assessment of the proposed framework is performed. In Chapter 4, the evolving edge-cloud computing architecture and the deployment models are described. The performance of performing communication, computing and intelligence tasks are evaluated on the state-of-the-art edge-cloud computing infrastructures. Chapter 5 presents the proposed IoT framework with an industrial application, i.e., the vertical plant wall system, and demonstrate how the system is enhanced with the edge-cloud computing paradigm. Chapter 6 showcases how novel machine learning and neural network-based methods are leveraged in IoT applications to achieve intelligence. In Chapter 7, a reference framework that can guide SMEs to achieve digital transformation as well as several instructive principles for digital solution design are presented. Chapter 8 summaries the included publications in the thesis and Chapter 9 concludes the thesis. 


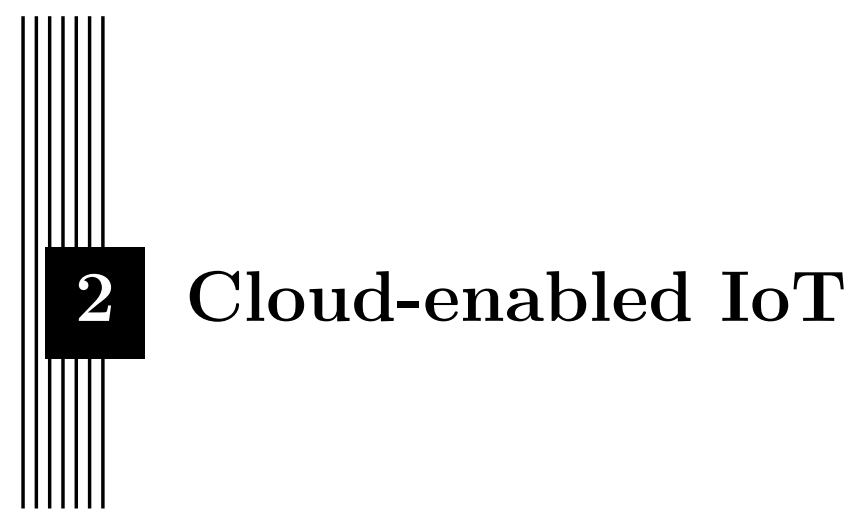

In this chapter, a data-centric IoT framework is presented. The cloud infrastructure and the local configuration are introduced and the uniform device and data models are described.

\subsection{Framework overview}

Figure 2.1 depicts the proposed data-centric IoT framework for massive IoT and broadband IoT applications. The framework consists of a local field part and a cloud platform. In the local field, sensors, actuators and gateways establish connectivity using WiFi, Thread and LoRa communication protocols to support local, personal and wide area networks (LAN, PAN and WAN). LAN enables Internet service to both low data rate and broadband applications, e.g., smart electronics and surveillance system. PAN supports low data rate and low power devices that are used within personal living space, such as building automation systems, wearables and e-health systems. WAN extends network infrastructure to a wide coverage, which can be adopted in farming, logistics, metering and smart city. IoT data that are collected from sensors installed in the field networks are continuously forwarded to the cloud via a WiFi access point. Alternatively, an edge device can be deployed in the local field to enhance local processing capabilities such as data cleaning and local computing. Low power sensors that are deployed in a WAN can send messages using the LoRa network, taking advantage of the low power consumption and long range communication features. 


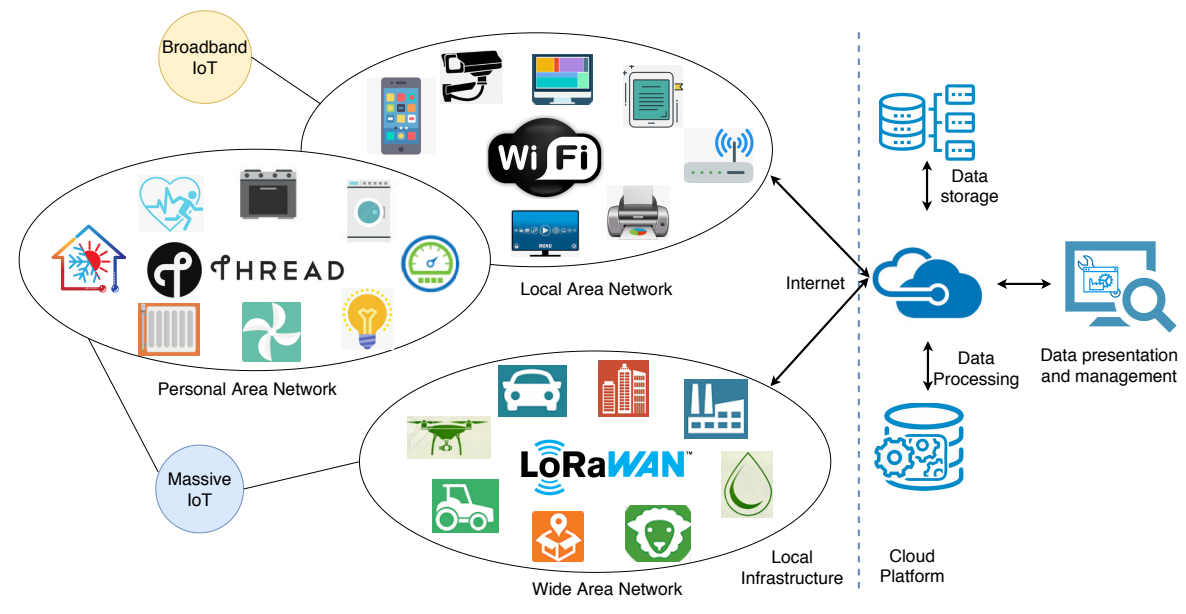

Figure 2.1: Overview of the data-centric IoT framework for massive and broadband IoT [12].

The cloud part is a collection of data processing, storage, and presentation and management components. An IoT Hub service functions as the core unit in the cloud, which is responsible for device provisioning, device identity management and data routing. Other peripheral services can be flexibly integrated into the IoT Hub infrastructure as plugins, depending on practical needs of applications. A cloud gateway is in charge of authentication and data routing for all incoming messages that are transmitted from field devices, before forwarding to further processing, storage and visualization services. A container-based web application is developed to realize real-time visualization, historic data display and administrative management functions for end users.

\subsection{Azure cloud infrastructure}

In the proposed framework, service connectivity in the cloud infrastructure is shown in Figure 2.2.

IoT Hub is the core in the framework, which offers fully-managed device provisioning, authentication, identity management through digital twins, and data ingestion and routing. IoT Hub supports secure and bidirectional communications with millions of IoT devices using three common protocols, i.e., Hypertext Transfer Protocol Secure (HTTPS), advanced message queuing protocol (AMQP), and message queuing telemetry transport (MQTT). It can directly connect to IP-capable devices and establish connections to low-power and resource-constrained devices, or devices with other protocols via a field gateway [13]. 


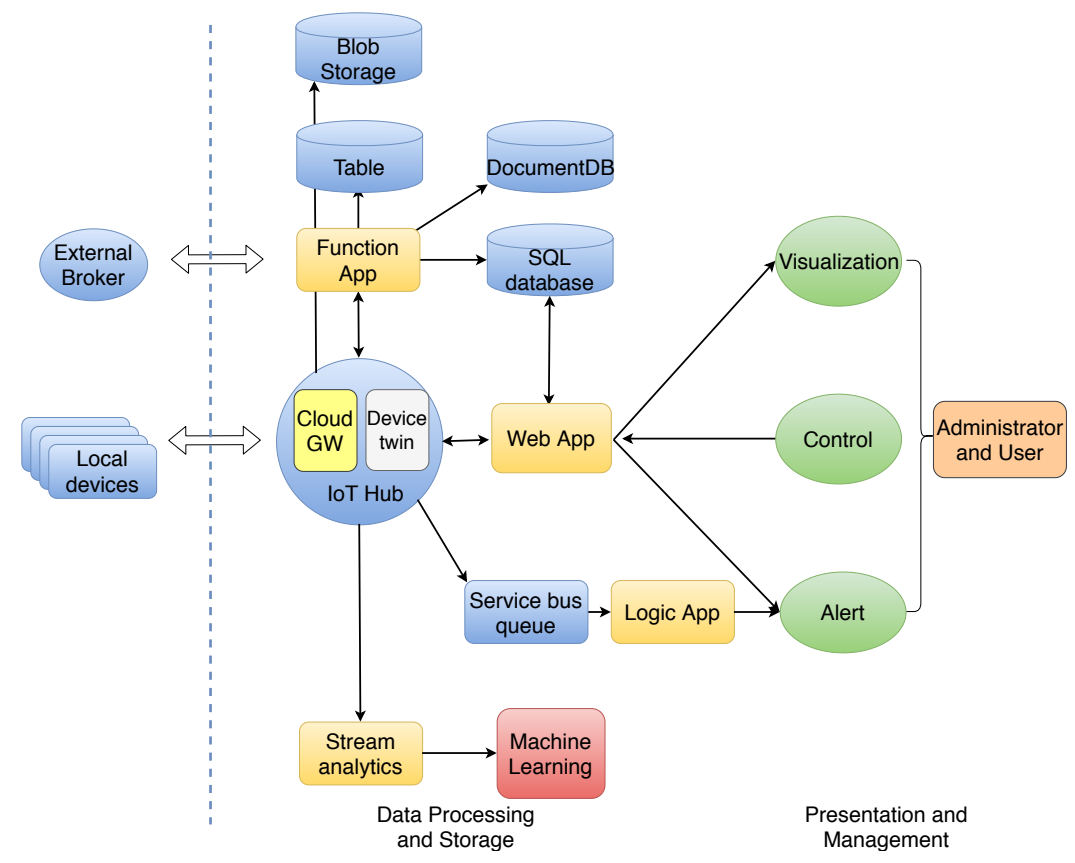

Figure 2.2: Cloud service connectivity of the data-centric IoT framework [12].

A series of peripheral services are seamlessly integrated into the IoT Hub to accelerate data analytics, storage or presentation of IoT telemetries.

Function service is a serverless service that enables direct execution of a function with a snippet of code while eliminating the necessity to develop a whole application. In the framework, function services serve as an adaptation layer between data input and storage services so that data from heterogeneous sources and locations can be adaptively stored to different databases according to their contents or properties.

Data storage service can vary depending on the type of received data. Four types of storage services are supported in this framework for IoT use cases. SQL database is used for relational data. DocumentDB features light weight, fast query speed and simple data format, e.g., JSON format. Table storage is reserved for table structured data. Blob storage is used for unstructured data such as uploaded video, image, raw data file, or device specific files.

Visualization service is built upon a web application-based humanmachine interface (HMI) in which customized visualization methods can be integrated in according to distinguished data sources.

Intelligence services is a service collection that covers data processing, analytic and logic units, and machine learning modules. For instance, stream analytic services together with built-in machine learning services can acceler- 


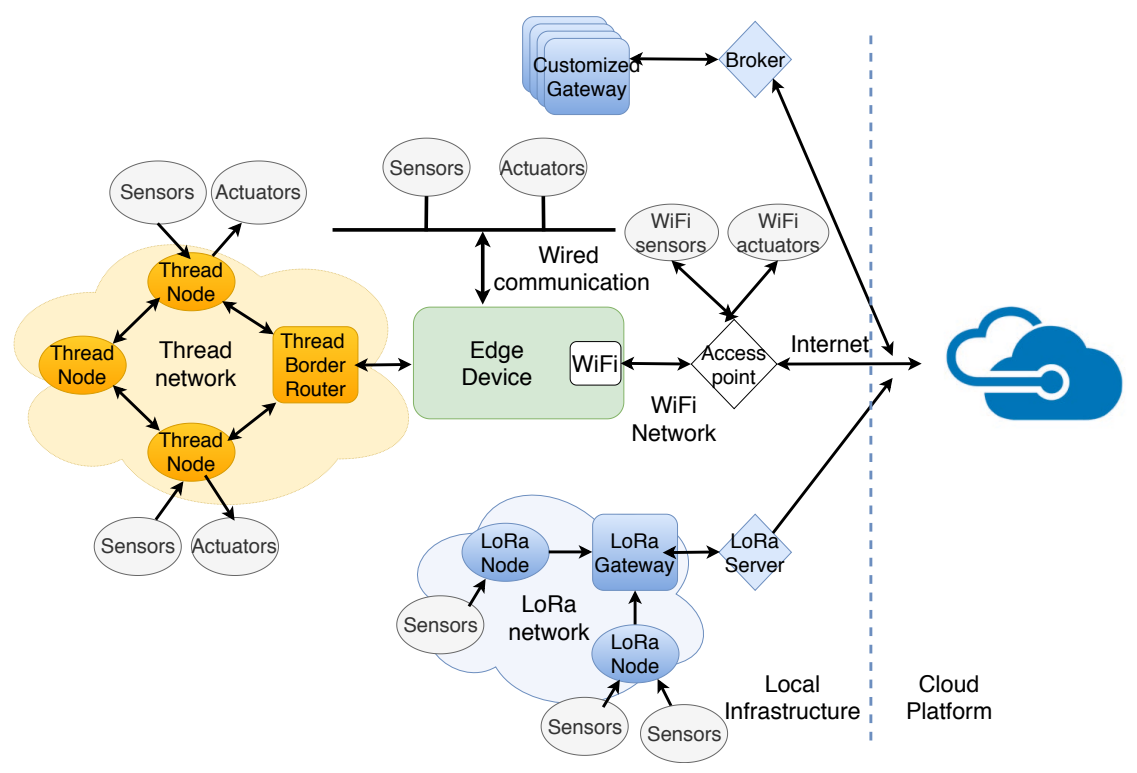

Figure 2.3: Local networks connectivity in the proposed IoT framework [12].

ate time series data analytics. A logic application can enable alert functions. More advanced and flexible machine learning models developed by users can be deployed together with the web application to realize customized intelligent functions.

\subsection{Local infrastructure}

The connectivity among local infrastructures is shown in Figure 2.3. The details are explained as below.

\section{Network connectivity}

In the framework, WiFi is the backbone network which connects local infrastructures to the cloud. WiFi capable devices can directly communicate with the cloud gateway using supported messaging protocols via an access point. In parallel, an edge device can bridge the message exchanges between resourcelimited devices and IoT Hub. To satisfy the low-power requirement, lightweight application layer protocols such as constrained application protocol (CoAP) and MQTT are leveraged to carry out bidirectional communications between the edge device and WiFi-enabled sensors.

Thread is introduced into the framework as a complement to the WiFi network. The connectivity between the Thread network and the edge device is supported by a Thread border router, from which IP traffics are adapted 
to Thread packets and vice versa. In the application layer, the interaction between a Thread device and the edge device is through the MQTT protocol and an MQTT broker, similar to WiFi nodes. Specifically, a lightweight MQTT protocol designed for sensor networks, namely MQTT-SN, is utilized to enable Thread devices to communicate in the same way as using MQTT, which largely improves the interoperability among the local field devices.

LoRaWAN is involved in the framework to provide services such as metering, tracking or other monitoring tasks, which operates at a wider scope than PAN and LAN. Considering that in industrial practice, a reliable downlink service in a LoRa network needs densely deployed gateways, the present framework only enables single directional up-link communication while neglecting down-link traffic. Due to the large scale deployments of gateways and superior compatibility between LoRaWAN servers and public cloud platforms, the LoRaWAN services are recommended to be built upon commercial LoRaWAN operators' network.

\section{Edge device}

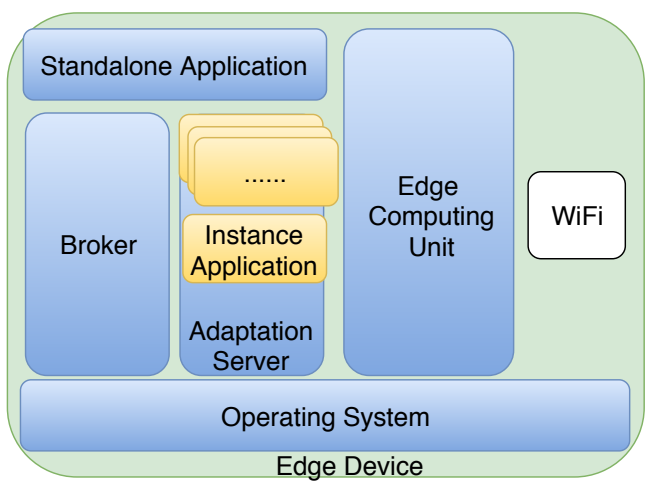

Figure 2.4: Architecture of the edge device used in the proposed IoT framework [12].

Figure 2.4 depicts the architecture of an edge device in the framework where message exchanges between devices and the cloud take place. The edge device is compromised of standalone applications, an edge broker, an adaptation server, an Internet module and an edge computing unit. An standalone application is an isolated process interacting with sensors and actuators that are directly connected to the edge device, which has its own identity in the cloud. The broker is a message server that delivers messages from publishers to subscribers so as to enable the interoperability between the edge device and Thread/WiFi devices. The adaptation server maintains a couple of application instances. Each of them represents an end device and has its own device identity object, which is always synchronized to the correspond- 
ing device digital twin in the cloud. The Internet module is to enable an communication channel between the edge device and the cloud platform, e.g., a WiFi interface. The edge computing unit is an enhancement to the edge device and brings processing capability closer to local infrastructures.

Additionally, an external broker is exploited in the framework to improve the compatibility with other communication protocols, e.g., legacy field network protocols that have been widely deployed. The external broker can be subscribed by sensory devices that continuously publish messages and a function application running in the cloud that periodically fetches messages from the broker. In this way, the data generated from legacy protocols can be conveniently inserted into the same database as other first class devices.

\subsection{Device model}

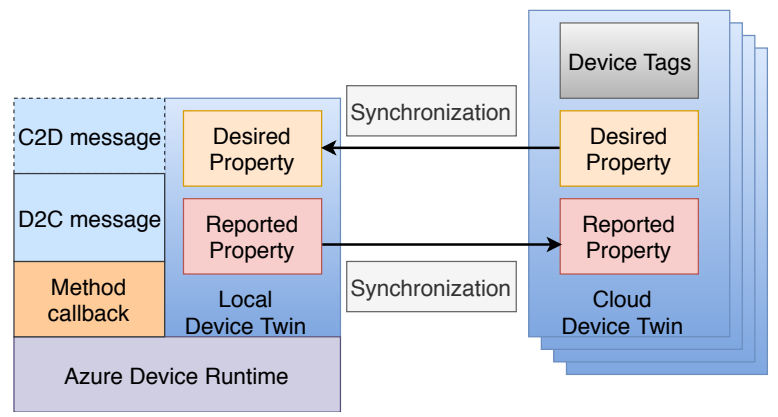

Figure 2.5: Device management model in the proposed IoT framework [12].

The proposed framework features a unified device management model, as shown in Figure 2.5, which is applied to all the registered devices. Upon device provisioning, all information related to a specific device such as device ID and authentication key, are stored in the cloud device identity registry, regardless of the hardware difference. Three patterns, i.e., property update, bidirectional message and direct method are covered by the device management procedure. Each device corresponds to a digital twin object, namely device twin, which is stored in the IoT Hub and in the local device. A device twin contains device tags, desired properties and reported properties. By synchronizing local desired properties to the cloud desired properties and updating the latest local reported properties to the cloud reported properties, the device twins are always consistent. Device to cloud (D2C) messages and cloud to device (C2D) messages enable telemetry and notification delivery. Direct method is an approach to instantly invoke local commands in the end device to execute. With the aforementioned device twin objects and three device management patterns, this framework can treat all devices with a single device model. 


\subsection{Data model}

The JavaScript Object Notation (JSON) encoding format are ubiquitously exploited by the industry and natively supported by many libraries and platforms [13]. In this framework, all the sensory messages and device twin objects are encoded in JSON to guarantee interoperability.

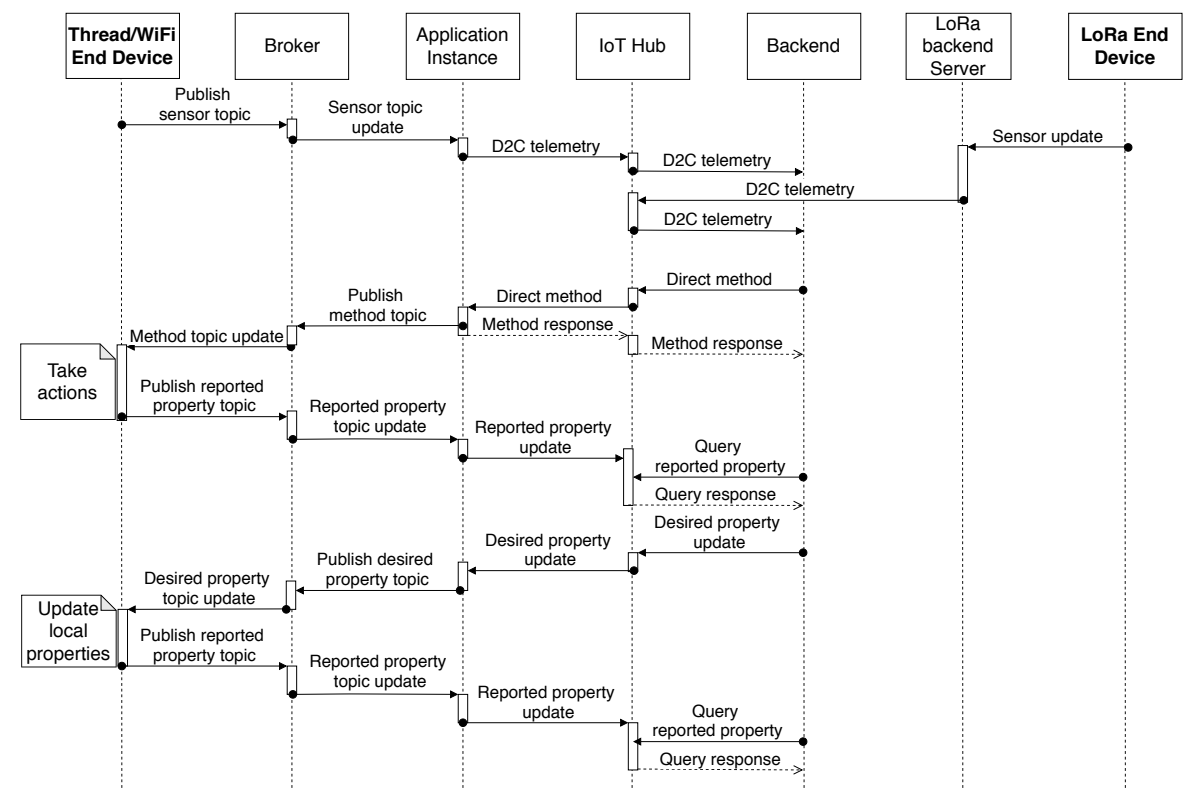

Figure 2.6: Data flow of Thread, WiFi, and LoRaWAN devices in the framework [12].

In this framework, the data flow between the cloud and end devices involves D2C telemetry, C2D direct method, desired property update, reported property update, and unstructured data upload. Figure 2.6 depicts the data flow of Thread, WiFi and LoRa nodes. A Thread or WiFi end device can interact with the corresponding application instance through the MQTT broker. Upon receiving a notification from the broker, the application instance constructs a D2C telemetry for the Thread/WiFi node and sends it to the cloud. Similarly, a direct method or desired property update is received by the application instance and then forwarded to the Thread/WiFi end device using MQTT broker-based notifications. Sensor data from a LoRa end device are relayed by a LoRa backend server, from where data are encapsulated into D2C messages and transmitted to the cloud.

Details of the framework and a reference implementation are presented in Paper I. 



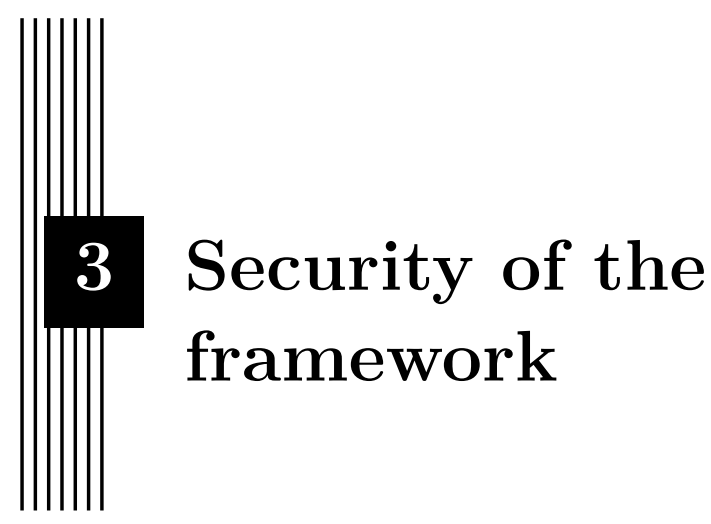

In this chapter, a security analysis taxonomy is proposed as a tool to evaluate security aspects and identify potential security flaws in field network communication of IoT applications. It is a significant complement to enhance the security considerations of the proposed IoT framework.

\subsection{Security analysis taxonomy}

Security is a major concern in IoT practice and always prioritized at the beginning of IoT solution design by practitioners. Figure 3.1 shows the proposed security analysis taxonomy intending for security assessment in an IoT field network, e.g., a building automation network (BAN) in a building automation system (BAS), which is a case of IoT applications in the context of smart building.

The proposed taxonomy considers security in two aspects, i.e., network security and device security, which are further divided into five phases of interaction that are detailed as follows.

Secure Commissioning Commissioning describes the process that starts from a device locates the correct network, authenticates itself to the authorized commissioner in the network, and is entrusted with network credentials, to a secure communication channel with other entities in the network is established. In order to achieve a secure commissioning procedure, security during network detection, authentication and the delivery of network secrets has to 


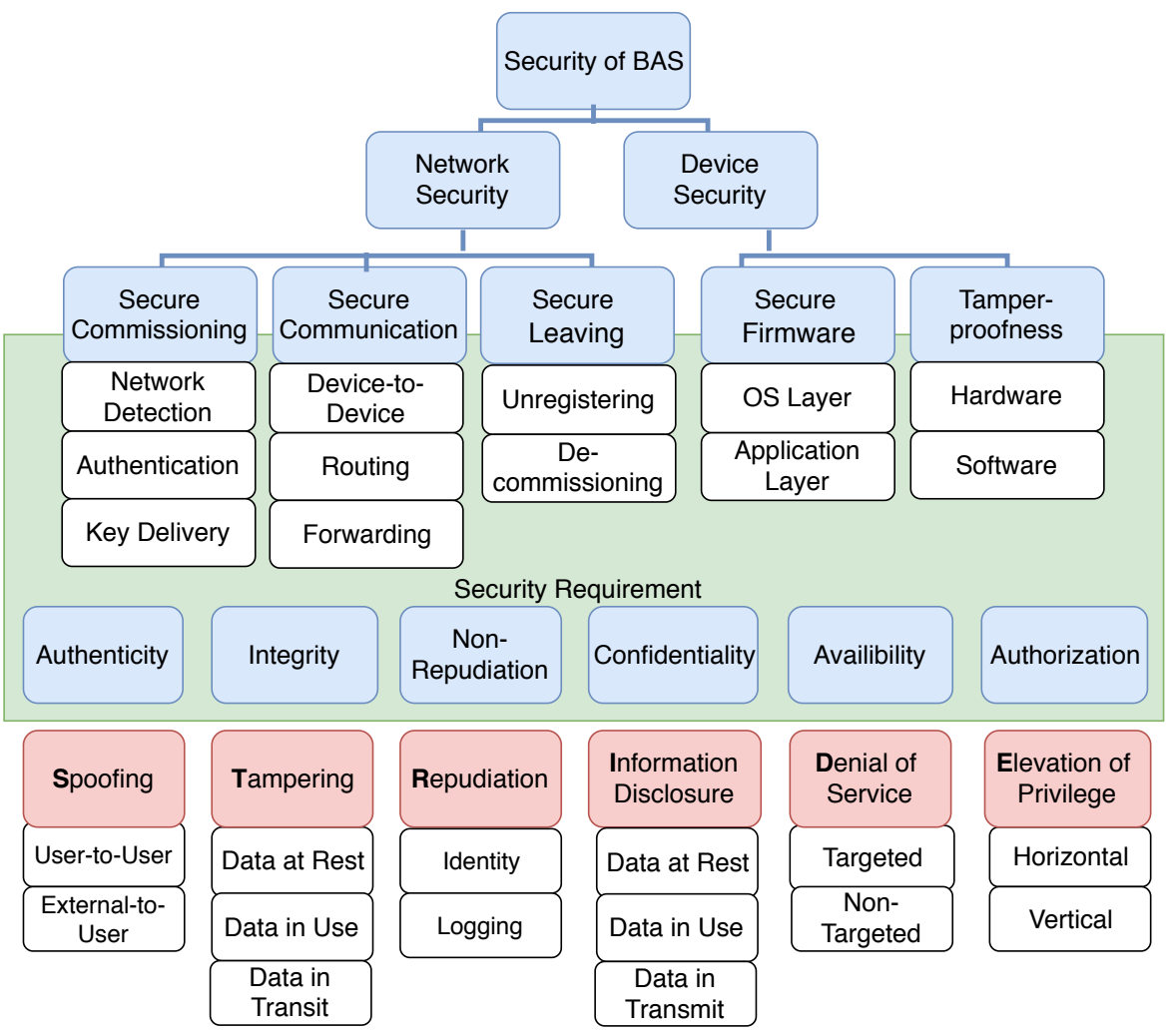

Figure 3.1: The proposed security analysis taxonomy for building automation system (BAS). It can be extended as a general tool for security assessment in IoT field networks [14].

be guaranteed. A network protocol has to protect the network from spoofing, man-in-middle and denial-of-service (DoS) attacks and should not leak any secret to unauthorized third parties.

Secure Communication A device performs given functions through communications over the established channel within a field network using the network credentials. In order to secure the communication channel, routing information exchange, message forwarding, and device-to-device data delivery should be secure.

Secure Leaving Once a commissioned device is requested to leave the attached network or to join another network, it has to be removed from the network (unregistering) and destruct sensitive information about existing network (de-commissioning) in a secure manner. Exposure of the network credentials can result in security violation of other devices within the network. 
Secure Firmware Firmware is stored in non-volatile memory and responsible for the realization of device functions. The firmware can be divided into a low level operating system (OS) that manages hardware resources, and high level applications that execute functional tasks. A compromise at any of these two levels leads to compromise of the device. Therefore, OS and application development, as well as firmware upgrades shall be secured.

Tamper-proofness A field IoT device needs to be protected from compromise through tampering. A compromised device can be used for obtaining network credentials or network data manipulation. Protection through physical isolation is not always applicable. Therefore, tamper protection needs to be implemented on the chips and the memory inside the devices, to protect the hardware and the software.

\section{Security Requirements}

The proposed taxonomy formulates security requirements for all of the aforementioned five phases, taking consideration of threats that are structured according to a refined STRIDE threat model and cover both active and passive adversaries. In the refined STRIDE model, spoofing by an internal and an external adversary is distinguished. Tampering and information disclosure attacks against data at rest, in use and in transit are considered. Repudiation attacks are considered in two aspects, i.e., towards identity management and logging. It should also be distinguished between targeted and non-targeted DoS attacks, and between horizontal and vertical elevation of privilege attacks.

For all five phases, security requirements in terms of authenticity, Integrity, Non-repudiation, Confidentiality, Availability and Authorization are evaluated so as to have a comprehensive analysis of the security level of a IoT field network. For detailed descriptions of the taxonomy, see Paper II.

\subsection{Security assessment of the framework}

\section{Security in field networks}

The security in IoT field networks is evaluated for Thread, WiFi and LoRaWAN respectively. Thread is designed with superior security consideration. The security mechanism covers the whole life cycle of a Thread device, ranging from a device discovers and joins the network to leaves the network. A datagram transport layer security (DTLS) session is established for device commissioning, which fulfills online and offline dictionary attack resistance, forward secrecy and known session security. All communications are protected 
by an AES-CCM security suite to guarantee confidentiality, integrity and authenticity. In Paper II, a thorough security analysis about novel network attacks towards Thread is presented, and security enhancement countermeasures to reinforce the security of Thread are also proposed.

A majority of the WiFi devices are secured with WiFi Protected Access 2 (WPA2) encryption method, which has been found vulnerable to key reinstallation attack (KRACK) [15]. The upcoming generation of WiFi protocol, 802.11ax, is equipped with an enhanced encryption method namely WPA3 [16] that can mitigate the flaws in WPA2 such as offline dictionary attack and KRACK attack, and enables forward secrecy to prevent old data being disclosed by later attacks. In general, the new WiFi release will bring security to a high level.

LoRaWAN supports end-to-end security. Two types of session keys are utilized to establish a secure channel between an end device and a LoRa application server. A 128-bit AES security key is associated to each LoRa device to enable device specific security. LoRaWAN implements integrity protection in a hop-by-hop manner, i.e., one hop over the air is guaranteed by LoRaWAN protocol and the other hop between the network and the LoRa server is protected by secure transport solutions such as HTTPS and VPNs [17].

\section{Security in the cloud}

In the cloud platform where IoT data are routed among services, the security is guaranteed by Azure security infrastructure which provides high levels of enhanced security, privacy, compliance, and threat mitigation practice [18]. IoT Hub adopts per-device authentication to guarantee security risks are isolated for separate devices. Each device key is bound to an access policy when it is generated, which regulates the authority to access a specific resource and greatly improves the security in authorization. Transport layer security (TLS) based handshake and encryption are employed in communications between IoT Hub and IoT devices, which is seen as the cornerstone of the security infrastructure of today's Internet society.

Detailed discussion about security of WiFi, LoRaWAN and the cloud platform can be found in Paper I. It is worth noticing that the proposed framework has taken up the advantages in network security, while implementation details regarding device security such as secure operating system, secure application layer, and hardware tamper-proofness shall be further considered. 


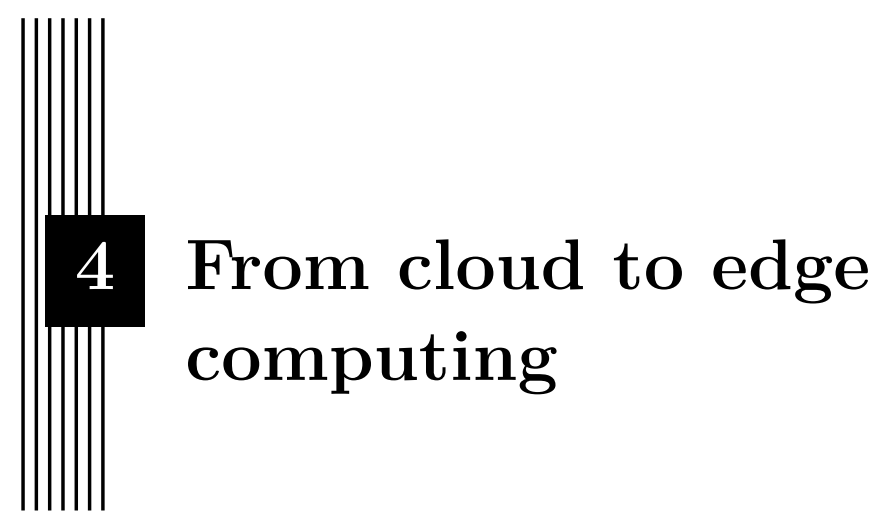

Recently, a tendency has been observed that cloud computing is evolving towards the edge-cloud computing paradigm in IoT applications. As a further study to the previously proposed IoT framework, the edge-cloud computing architecture can be adopted to enhance the capabilities of the framework in communication, computing, and intelligence. Therefore, the performance of the edge-cloud computing stack, especially the containerization-based virtualization technology, shall be investigated. In this chapter, a comprehensive performance evaluation on the full stack of edge-cloud computing is conducted and presented, which highlights the capability and promising future of the container technology in the edge-cloud computing era.

\subsection{Edge-cloud computing stack}

\section{Architecture}

Fig. 4.1 illustrates a containerization-based edge-cloud computing architecture. In the edge unit, containerized applications are managed by a container runtime. These modular applications can be remotely created, upgraded and destroyed in an elastic manner with low overhead while the procedure can be fully managed with container orchestration tools such as Kubernetes [19]. Similar to the role of IoT Hub in the cloud, an edge hub module is deployed at the edge device to maintain traffic in the edge, including bidirectional communication between field devices and the cloud platform, and message routing 


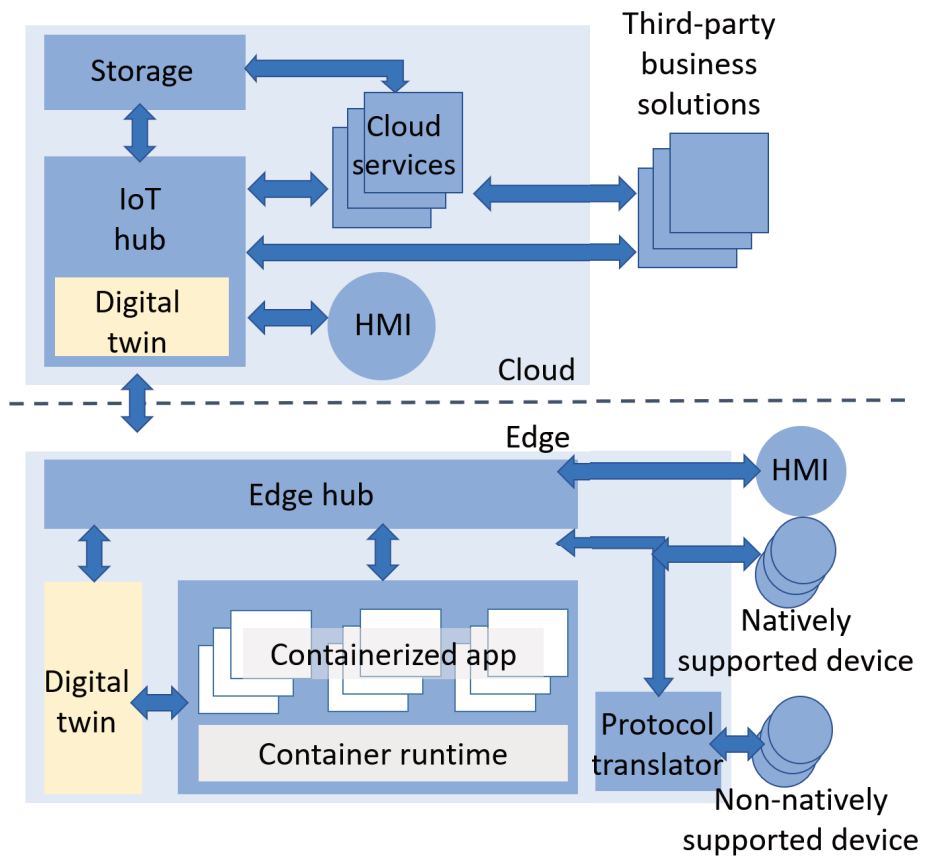

Figure 4.1: Architecture breakdown of the edge-cloud computing paradigm for containerization-based industrial IoT applications [10].

among containerized applications. Field devices can initiate communications to the edge hub so as to get services from edge applications. The communication is established either using natively supported protocols such as the MQTT protocol or through a protocol translator. This illustrated design has become the de facto architecture of edge-cloud computing that is put into industrial practice, e.g., Microsoft Azure IoT Edge [20], Amazon Greengrass [21], IBM Edge Application Manager [22], and Huawei KubeEdge [23], and is a promising approach to deploy industry artificial intelligence and IoT (AIoT) applications [24].

\section{Deployment models}

Three typical models that describe the connectivity between the field devices and the edge and cloud platforms can be utilized to deploy applications.

\section{Device-cloud model $(D-C)$}

In the device-cloud model, field devices establish a direct connection to the cloud and neglect the edge infrastructure. The field device only takes advantage of the networking capability which enables Internet access the edge de- 
vice. Communications between the field device and cloud services are straightforward within an established session. This model stems from the traditional cloud computing paradigm in which the edge device only functions as a router.

\section{Device-edge-cloud model ( $D-E-C)$}

The device-edge-cloud model originates from the device-cloud model but can enhance reliability of applications. The edge hub aggregates traffics from the field devices, which are then forwarded to the cloud with a multiplexed communication channel to reduce bandwidth. In case the connection to the cloud is intermittently lost, traffics generated by field devices can temporarily be held at the edge device till the connection to the cloud recovers.

\section{Device-edge model $(D-E)$}

In the device-edge model, more responsibilities are relocated to the edge device, i.e., both communication and computing take place at the edge device. In this model, field devices can directly communicate to and get responses from edge-native services, which greatly benefits time-critical applications or IoT use cases when the cloud is not reachable.

\subsection{Performance evaluation of edge-cloud architecture}

\section{Evaluation methodology}

Full stack round trip time (RTT) is used to characterize the latency feature for a typical edge-cloud architecture, which is more meaningful to depict the holistic performance and provides insightful knowledge to the solution design. Fig. 4.2 illustrates the measurement of the full stack RTT in the aforementioned three deployment models. With full stack RTT as the metric, several parameters that can influence the system performance are benchmarked, i.e., the message sending interval, the payload size, the network bandwidth and the amount of concurrent devices. The full stack RTT is calculated as follows.

$$
R T T_{\text {FullStack }}=T_{\text {ClientReceive }}-T_{\text {ClientSend }}-T_{\text {Server }}
$$

where

$$
T_{\text {Server }}=T_{\text {ServerSend }}-T_{\text {ServerReceive }}
$$

In the application layer, the MQTT protocol is utilized in the evaluation, and the raw socket implementation is also evaluated as a comparison.

Processing capability is proposed to measure computing and intelligence capabilities of the edge-cloud computing architecture in order to explore the system limitation and to serve as a guideline for solution design in 


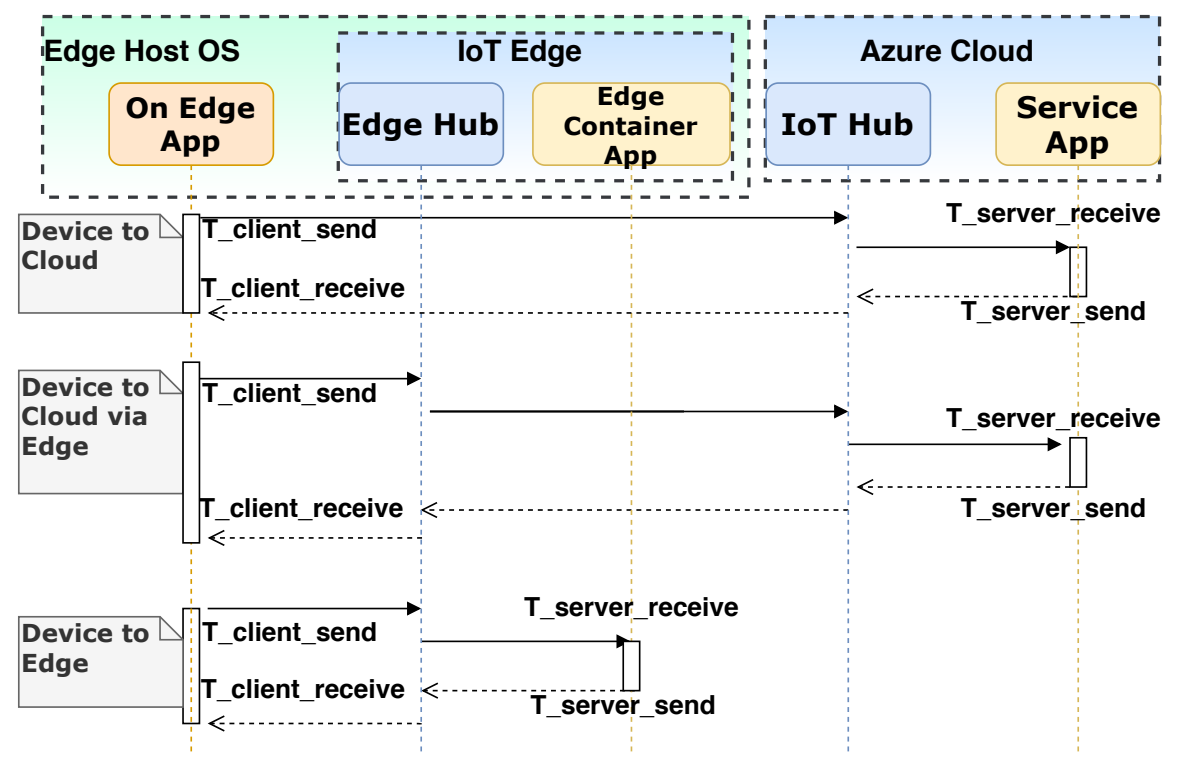

Figure 4.2: The data flow of three benchmarking cases. (1, 2) Devicecloud/device-edge-cloud: an application directly connecting to the cloud / connecting to the cloud via the Edge hub infrastructure, and getting a response from a service application hosted in the cloud. (3) An application connecting to the Edge hub and getting a response from a containerized application hosted at the edge [10].

AIoT applications. In the evaluation, training and prediction tasks for machine learning and neural network models are adopted for stress test while the execution time, CPU load and memory utilization are recorded for comparison.

\section{Evaluation platform}

Microsoft Azure IoT Edge is utilized as the edge infrastructure, which offers a container-based edge computing framework that enables customized services to be deployed to the edge as containers. An edge agent module is responsible for instantiating containerized applications and monitoring application health while an edge hub module handles message communication and routing. A BCM2837B0 system on chip (SoC) based Raspberry Pi 3B+ board is used as the hardware platform, which runs a Raspbian Linux operating system. As for the cloud, an IoT Hub service and a virtual machine (VM) are subscribed in the Azure cloud to provide message ingestion and to run service applications, respectively. 


\section{Highlights of experiment results}

Figure 4.3 gives a visual comparison between the edge host OS and the edge container with regard to resource utilization of executing a long short-term memory-encoder-decoder (LSTM-ED) neural network model. The result suggests that, compared to running on the host OS, the container-based virtualization does not introduce a considerable performance downgrade but offers additional flexibility and scalability to the deployment of machine learning applications, which complements the computing and intelligence features in the edge-cloud computing paradigm.

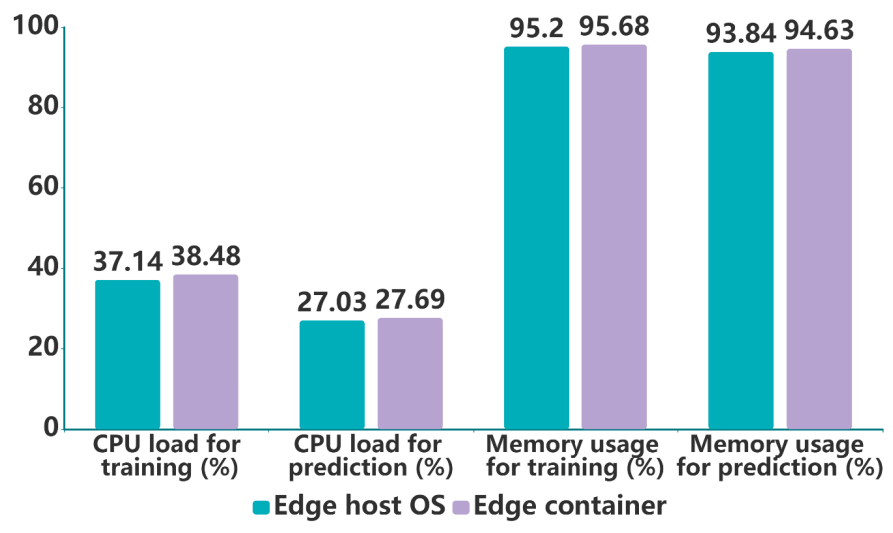

Figure 4.3: A comparison of resource utilization between the edge host OS and the edge container with regard to executing the LSTM-ED model [10].

The key findings in the evaluation are reflected in Figure 4.4 and listed as follows.

- When testing on existing containerization based edge-cloud computing infrastructure, an average full stack RTT above $100 \mathrm{~ms}$ is observed, regardless the service is located to the cloud or the edge.

- The minimal message sending interval is $200 \mathrm{~ms}$ to guarantee time sensitivity and determinism in the evaluation, which is far worse than the performance promised by the cloud supplier. This indicates the cloud industry is not prepared to enter the industrial automation sector but remain in the massive IoT domain.

- A non-negligible degradation of system performance is observed when 20 concurrent nodes are connected to the edge, which is far behind the industry automation requirement in which up to 10000 nodes can be deployed. 


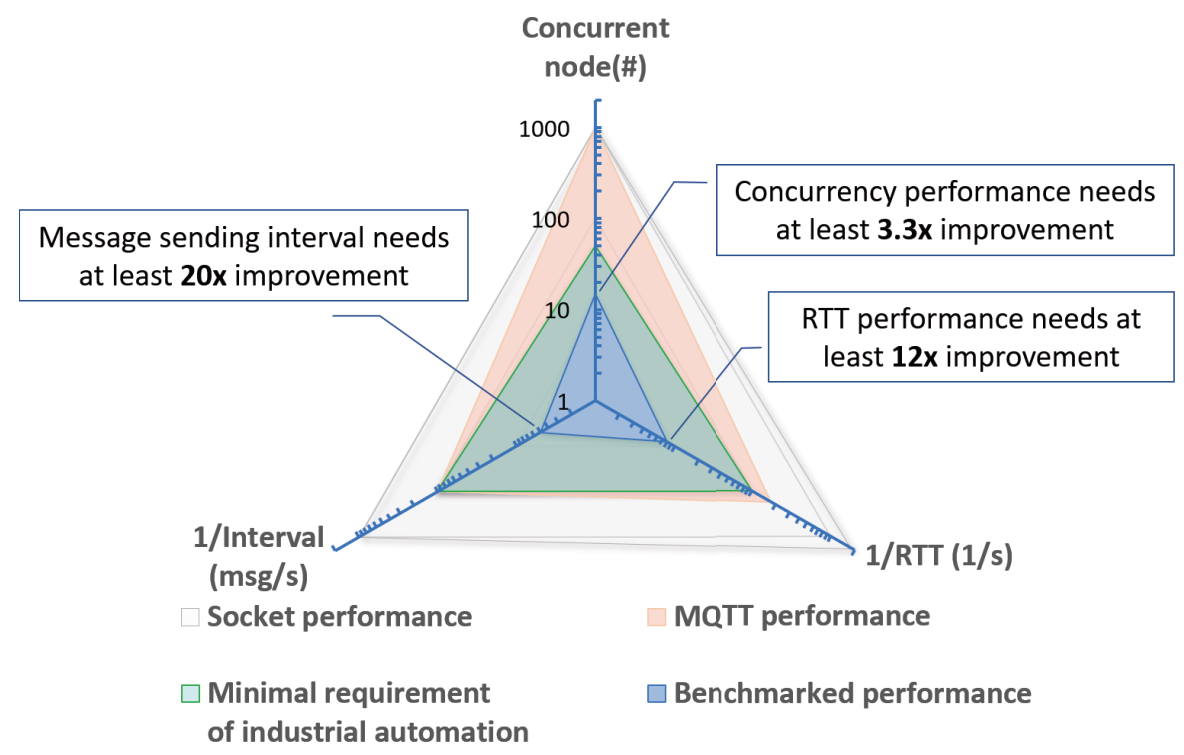

Figure 4.4: Performance gaps between the benchmarked edge-cloud infrastructure, the minimal requirement for industrial automation, and the performance of raw MQTT/Socket protocols are rather large. (Note logarithmic scale is used.) Current edge-cloud infrastructure needs to improve at least 3.3, 12 and 20 times in terms of concurrency, RTT and message sending interval to fulfill industry automation needs [10].

- Variation of network bandwidth has little impact to system performance whereas a payload below 10 kilobytes can guarantee the quality of service, which is sufficient for many industry automation use cases.

- The MQTT protocol and the TCP/IP stack are able to satisfy the demanding latency needs for time-critical tasks in IIoT, which achieve 5.7 ms and less than $1 \mathrm{~ms}$ full stack RTT, respectively. The bottleneck that limits the latency performance lies in the edge-cloud computing infrastructure, which shall be largely optimized.

- The performance of current edge-cloud infrastructure shall be improved by at least 3.3, 12, and 20 times in concurrency, RTT and message sending interval, respectively, in order to reach the minimal requirement of industry automation.

- Compared to the host OS, container-based virtualization does not bring noticeable performance degradation in terms of communication, computing and intelligence. The container technology is a promising approach in the edge-cloud computing paradigm to deploy industrial applications. It is able to execute machine learning tasks at the edge. 
However, the execution efficiency and resource utilization suggest the cloud shall be prioritized for heavy task load.

The detailed experiment configurations, statistic presentation and discussion of the evaluation results can be found in Paper III. 



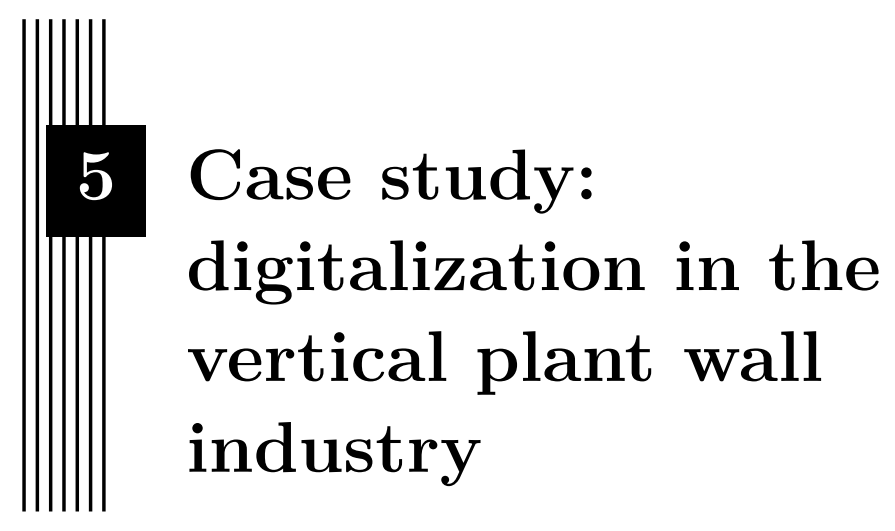

In this chapter, a real industry application, i.e., the vertical plant wall system, that undertakes IoT-enabled digital transformation leveraging the proposed IoT framework is presented. The technological evolution from cloud computing to edge-cloud computing in this application is highlighted.

\subsection{Background}

As shown in Figure 5.1, a vertical plant wall system is a vertical wall with diverse types of vegetations growing on the surface, which integrates growing medium, irrigation, lighting, and ventilation systems to support plants growing. Through evaporation, air purification and water retention effects, a plant wall can improve indoor environments significantly. For example, [25] shows that vertical plant walls can effectively purify air pollutants like particulate matter (PM) and volatile organic compounds (VOC) and meanwhile stabilize $\mathrm{CO}_{2}$ concentrations so as to improve human comfort and work productivity and reduce energy use.

The company Vertical Plant Systems AB (VPS) [26] is a plant wall manufacturer and supplier located in Sweden. Plant wall sales are limited to public spaces such as shopping malls, universities and museums due to several realistic challenges. Plant walls need regular plant care by staffs with relevant expertise, which is costly, time-consuming and labor-intensive. Geographically, it restricts VPS to distribute plant walls to remote places. Therefore, a digital solution that can remotely monitor the plant walls and automate 


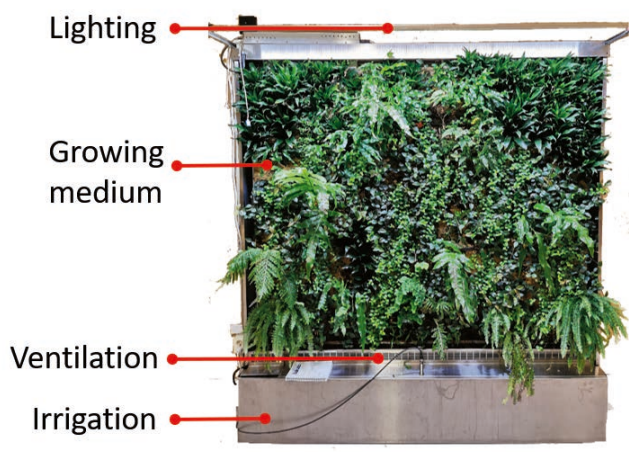

Figure 5.1: An example of a vertical plant wall system.

the plant care is demanded by VPS. A collaborative project between VPS and Linköping University was initiated to accelerate the digitalization and a detailed account of the procedure is described below.

\subsection{System overview}

Figure 5.2 shows an overview of the system for vertical plant walls. The system consists of a local monitor and control part, and a cloud server part. In the local part, a microcontroller is used to read environment sensors due to its deterministic timing, whereas a microprocessor with non-deterministic timing and an operating system is employed to execute complicated tasks. The sensor readings fetched by the microcontroller are periodically sent to the microprocessor upon query. Actuators, i.e., the water pump, the LED light and the fan installed in the plant wall, are under autonomous control by the microprocessor according to defined time schedules. These settings are locally stored as device properties and have an identical copy recorded in the digital twin in IoT Hub. By updating the digital twin, administrators are able to manage the schedule and control the irrigation, lighting and ventilation systems in the plant wall. A WiFi module is integrated with the microprocessor chip to enable communication with the remote cloud via any available access points that are common Internet infrastructures deployed in modern buildings. The local system can work in both online mode and offline mode, depending on if the connectivity to the cloud is alive. Switching between the two modes is transparent to users.

The cloud part is built up on the infrastructure of the Azure cloud platform. Periodic messages sent from the local part will be processed by IoT Hub and then routed to different services, such as storage and visualization. A containerized web application host in Azure cloud can provide real-time and historical data visualization. Through the interface of the web application, 
administrators and users are able to manage plant walls by directly invoking actuators or updating time schedules.

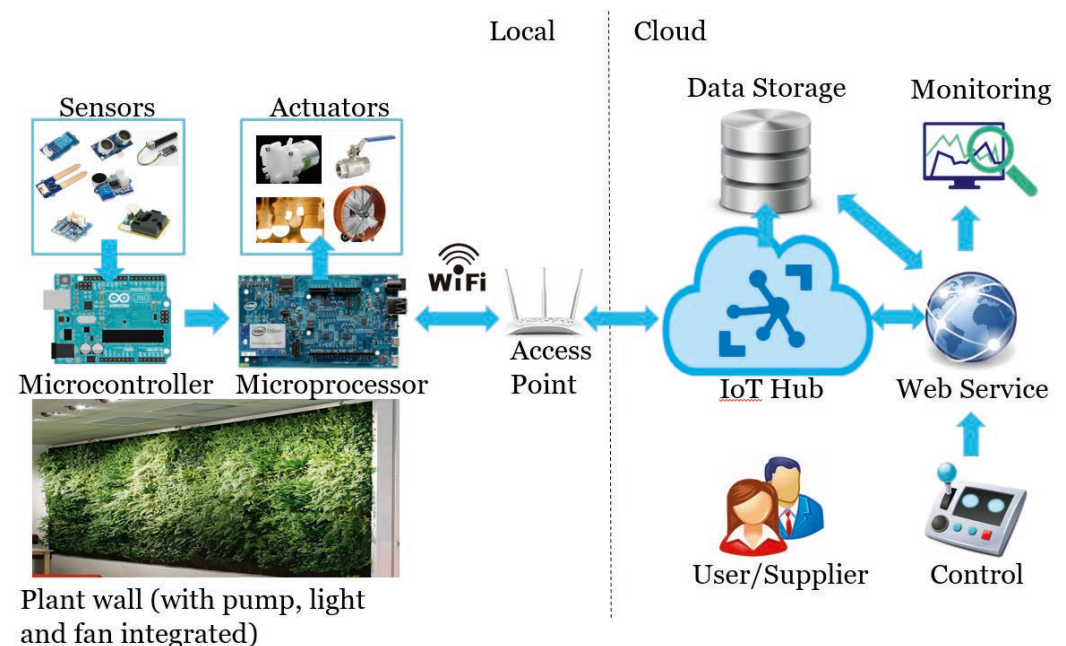

Figure 5.2: System overview of Azure cloud and IoT-based remote monitoring and management system for plant walls [27].

\subsection{Implementation}

\section{Hardware}

Figure 5.3 depicts the hardware connectivity of the proposed solution. The microprocessor deployed in the local part is responsible for transmitting sensor data to and receiving down stream messages from the cloud and executing plant care functions. In the solution, an Edison system on chip (SoC) module is selected as the microprocessor, which integrates a dual core Intel Atom CPU and on board WiFi module. The Edison board features rich interfaces such as UART, I2C, SPI and I2S as well as abundant GPIO pins for digital communication. A modern embedded Linux distribution, Yocto Linux, is leveraged as the operating system on Edison. As for the microcontroller, the Arduino Uno board is adopted to provide interfaces to sensors and actuators. It is based on the Atmega 328 chip and features six analog input pins and 14 input/output digital pins, of which 6 can provide PWM output capabilities. A series of sensors and actuators are deployed to the plant wall and the details of the models are presented in Paper IV.

\section{Software}

The program running on the Arduino board periodically fetches all the sensor status in turn and the results are repeatedly stored in a local buffer. Once it 


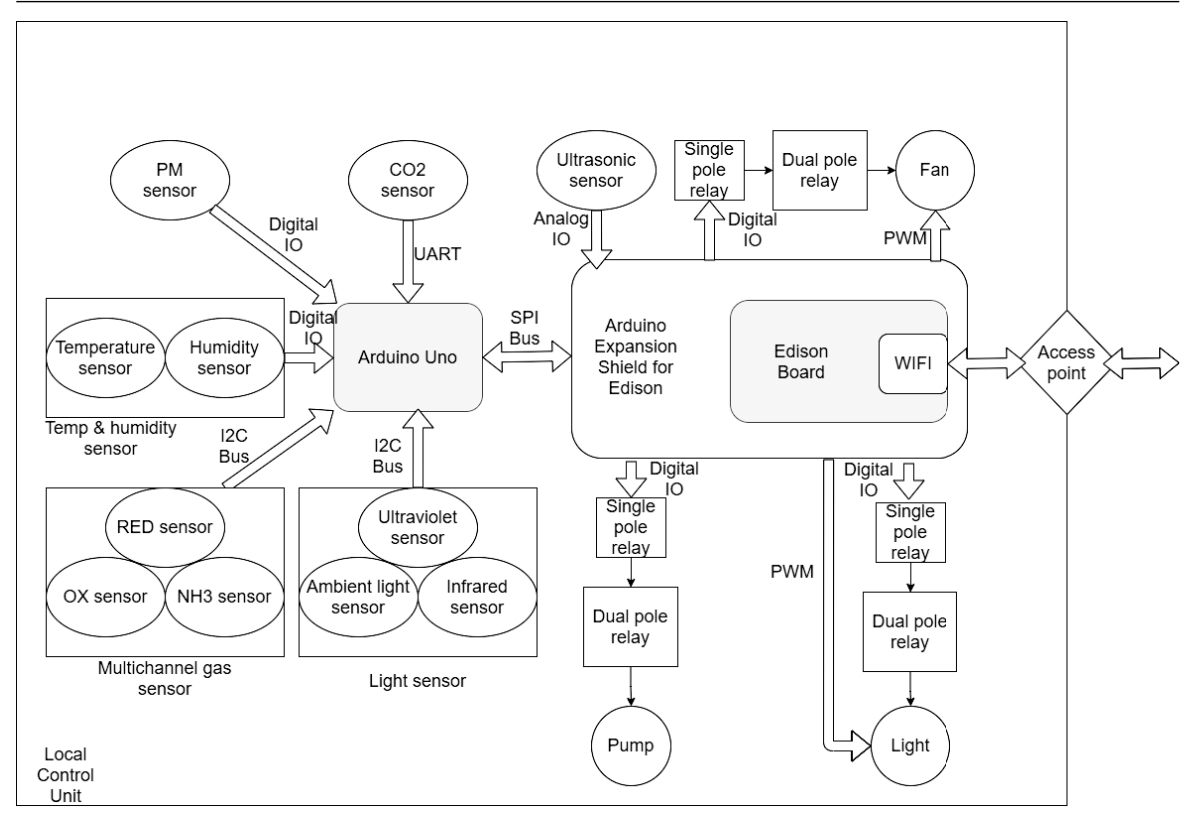

Figure 5.3: Hardware connectivity of the proposed solution for vertical plant walls [27].

\begin{tabular}{|c|c|c|c|}
\hline \multirow{19}{*}{ Properties } & \multirow{9}{*}{$\begin{array}{c}\text { Desired } \\
\text { Properties }\end{array}$} & Desired Interval & \\
\hline & & \multirow{3}{*}{ Desired Fan } & FanOnTime \\
\hline & & & FanOffTime \\
\hline & & & FanSpeed \\
\hline & & \multirow{3}{*}{ Desired Lamp } & LampOnTime \\
\hline & & & LampOffTime \\
\hline & & & Brightness \\
\hline & & \multirow{2}{*}{ Desired Pump } & PumpTime \\
\hline & & & PumpPeriod \\
\hline & \multirow{10}{*}{$\begin{array}{l}\text { Reported } \\
\text { Properties }\end{array}$} & Reported Interval & \\
\hline & & \multirow{3}{*}{ Reported Fan } & FanOnTime \\
\hline & & & FanOffTime \\
\hline & & & FanSpeed \\
\hline & & \multirow{3}{*}{ Reported Lamp } & LampOnTime \\
\hline & & & LampOffTime \\
\hline & & & Brightness \\
\hline & & \multirow{2}{*}{ Reported Pump } & PumpTime \\
\hline & & & PumpPeriod \\
\hline & & Reboot Number & \\
\hline
\end{tabular}

Figure 5.4: Plant wall properties are recorded in the digital twin and stored in both local device and the cloud [27].

is interrupted by the Edison board, the latest data are fed back to Edison via SPI bus. The application running on the Edison board is developed based on 
the MRAA library and the Azure-IoT-SDK. The application can operate in online and offline two modes to guarantee reliability when a loss of Internet is experienced. Major tasks of the application include control of actuators, query of sensor values, message update, digital twin synchronization, and execution of direct methods. Figure 5.4 shows an example of the properties associated with the plant wall that are store in the digital twin object in the device and the cloud. Administrators can remotely update the desired properties to adjust the control of actuators and get the real-time status by verifying reported properties. Additionally, commands can be sent to the device using direct methods to guarantee immediate execution of tasks.

\section{Cloud part}

The cloud takes charge of data processing, storage, and visualization functions, based on a collection of Azure cloud services that include IoT Hub, Function Application, SQL Database, Storage Service, Web Application, and Logic Application. IoT Hub in the core of the cloud solution, which performs device management and message routing. Each plant wall device authenticates itself to IoT Hub using a device-specific connection string and keeps track of the corresponding digital twin object to get real-time updates. Device to cloud messages containing sensor data are routed to function applications and then inserted to the SQL database in a structured manner while images and files are stored to a blob storage service. A web application is developed to provide human-machine interface for users. Both live stream and historical data are displayed, and three visualization methods, namely Line graph, Stacked Area graph, and Horizon graph, are utilized to better reflect the patterns and relationships between time series sensor data.

The details of the implementation are presented in Paper IV.

\subsection{Evolving to the edge-cloud architecture}

Catering to the industrial tendency, the cloud enabled IoT solution for vertical plant walls is migrated to an edge-cloud architecture. Tasks are partitioned and encapsulated into microservices leveraging the container technology. Depending on distinct performance requirements, these microservices are distributed across the edge-cloud computing infrastructure. The block diagram of the edge-cloud architecture of the upgraded solution is shown in Figure 5.5. Compared to the previous solution, an edge infrastructure that is based on Azure IoT Edge and Moby Container Engine is deployed to the device, in which an EdgeAgent module is used for modular application management and an EdgeHub module takes care of message routing among modules, devices and the cloud.

The detailed partitioning strategy is presented in Table 5.1. The system functions are partitioned into microservices that are distributed across the 


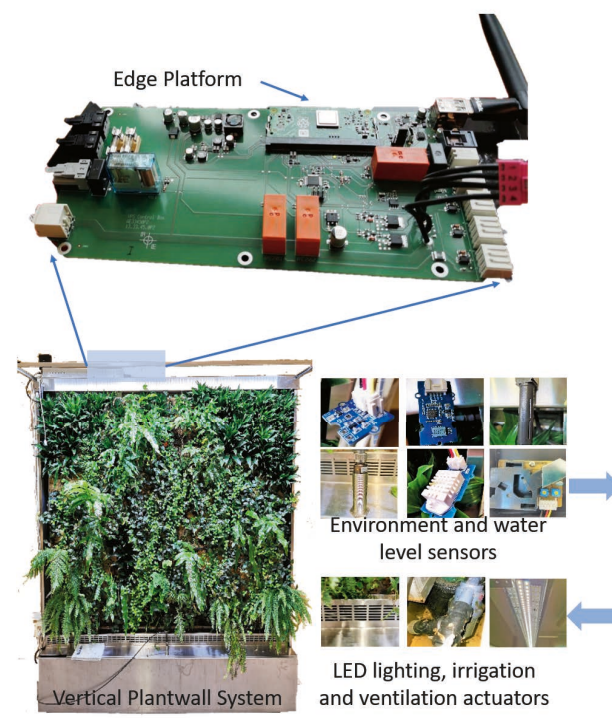

(a) Hardware setup

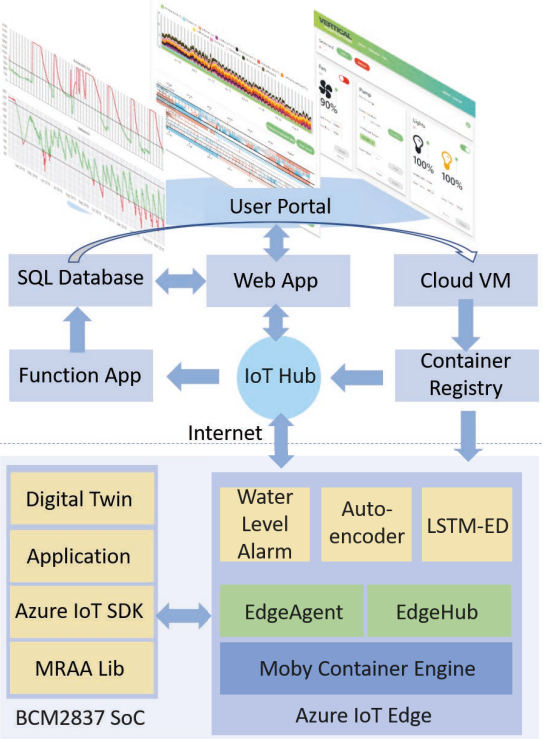

(b) Edge-cloud computing architecture

Figure 5.5: (a) Hardware setup of a vertical plant wall system that equips with sensors, actuators, and a self-implemented edge platform. (b) The block diagram of the edge-cloud computing architecture for the vertical plant wall system. A partitioning strategy is adopted to break down system tasks into microservices which are deployed to the edge host OS, edge containers and the cloud [10].

Table 5.1: Static partitioning of a vertical plantwall system.

\begin{tabular}{|c|c|c|c|}
\hline Function & Microservice & Performance Requirement & Platform \\
\hline $\begin{array}{l}\text { Sensor update } \\
\text { Actuator control }\end{array}$ & $\begin{array}{l}\text { Local application for sensor } \\
\text { reading and actuator control }\end{array}$ & $\begin{array}{c}\text { Latency tolerable, offline capability, } \\
\text { hardware accessibility }\end{array}$ & Host OS \\
\hline Water level alarm & $\begin{array}{l}\text { Containerized water level } \\
\text { alarm and control service }\end{array}$ & Latency $<200 \mathrm{~ms}$, offline capability & \multirow{2}{*}{$\begin{array}{l}\text { Edge container } \\
\text { engine }\end{array}$} \\
\hline Anomaly detection & $\begin{array}{c}\text { Containerized auto-encoder model } \\
\text { Containerized LSTM-ED model }\end{array}$ & Online learning capability & \\
\hline Data storage & SQL database & High scalability, massive storage capacity & \multirow{3}{*}{ Cloud platform } \\
\hline Model training & VM and Container registry & Powerful computing capability & \\
\hline Human-machine interface & $\begin{array}{l}\text { Web application for } \\
\text { visualization and interaction }\end{array}$ & Remote accessibility & \\
\hline
\end{tabular}

edge-cloud computing infrastructures according to their performance requirements [10]. For example, in such a system, the sensor update and remote operation of actuators are latency tolerable but shall have offline capability and hardware accessibility, therefore are deployed to the host operating system as native applications to enable interfacing with hardware and communication with the edge infrastructure. In case of a water leakage, the pump shall be shut down in both online and offline modes with a minimal latency, therefore the water level alarm function is deployed as a containerized module 
in the edge. Two machine learning based anomaly detection modules using the auto-encoder and LSTM-ED models are also containerized and deployed to the edge. The periodically updated sensor data are routed to all three modules to get processed. The details of the models are presented in Paper V. The edge-cloud infrastructure applied to the vertical plant walls is described in Paper III. 



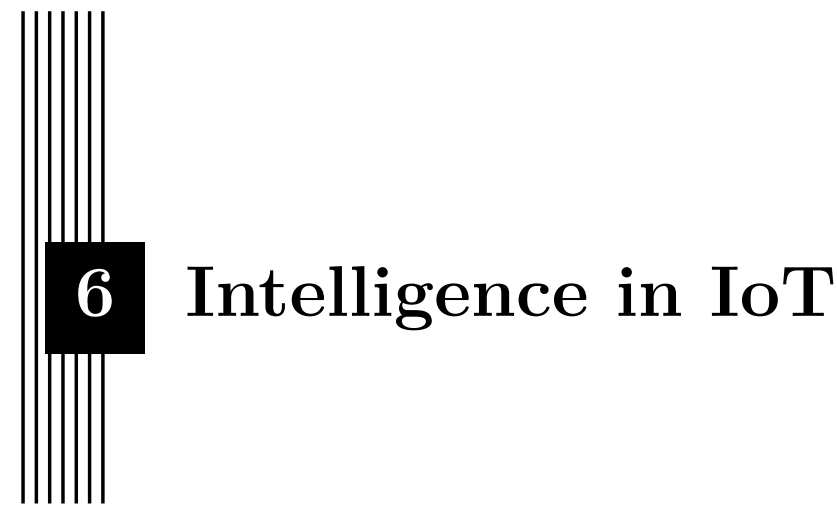

The ultimate goal of IoT is to extract intelligence from massive data and to achieve improved efficiency derived from the intelligence. This chapter showcases how to leverage machine learning (ML) and artificial intelligence (AI) in IoT applications with a practical example, i.e., anomaly detection for indoor climate control based on collected environmental sensor data, to highlight the added value enabled by IoT data.

\subsection{Background}

In the previous chapters, with the proposed IoT framework, a remote monitoring and management system for vertical plant walls has been achieved. Various environmental sensors deployed to the system enable real-time tracking of indoor climate. A further step is to achieve intelligence from the indoor climate data that are collected from massive deployed sensors. In this regard, machine learning can be leveraged to process and extract hidden information from vast data accumulated from indoor climate that can hardly be analyzed with human efforts. Accelerated by cloud computing, novel machine learning techniques such as deep learning and neural networks that demand powerful computation can be deployed to building environments in a variety of applications so as to achieve optimal building operations [28], energy consumption [29] and human comfort [30], etc.

Based on the established vertical plant wall platform, novel machine learning methods, specifically neural networks, are utilized to dedicate to anomaly 
detection tasks for indoor climate control so as to take full advantage of IoT data and boost the intelligence in indoor climate control. This transdisciplinary study also showcases how evolving researches in isolated disciplines such as IoT and machine learning can be employed to counteract the obstacles and accelerate the problem-solving and solution development in the research field of building environment.

\subsection{Anomaly detection for indoor climate control}

In this application, two categories of anomalies appeared in indoor climate data shall be identified, i.e., point anomalies and contextual anomalies. A point anomaly is a single outlier value in time series data, which can be caused by software error, hardware noise, or temporary interference in measurement. Contextual anomalies refer to a series of data values that inappropriately appear in a time period, although individually they are within the normal range of an indoor environment. Point anomalies can bring bias to further data processing while contextual anomalies indicate abnormal interference to the indoor climate, thus shall be detected to trigger relevant reactions.

\subsection{Experiment}

\section{Methodology}

In the experiment, a series of linear regression models are evaluated. Additionally, neural networks based models, e.g., artificial neural network (ANN), autoencoder (AE), recurrent neural network (RNN) such as long short-term memory (LSTM) network, and ensemble models like long short-term memoryencoder-decoder (LSTM-ED), are also benchmarked to get a model with the optimal performance in anomaly detection of indoor climate data. Those models are classified into two groups, i.e., prediction- and pattern recognitionbased methods. As shown in Figure 6.1, for prediction-based methods, a series of data points within an observation window are used as features that are fed into the model, and the next consecutive data point is the prediction target, which results in a one-step ahead prediction. For pattern recognition-based methods, features are prepared in two cases, which result in a sliding window and an overlapped sliding window dataset. In both cases, the targets are the same as the input features.

As for metrics, mean absolute error (MAE) and mean squared error (MSE) are utilized to estimate the prediction accuracy of models. Due to the nature that anomaly detection is a classification problem, receiver operating characteristic curve (ROC) is adopted as a graphical method to interpret the performance of classification models. Besides, the area under the ROC curve (AUC) metric is employed to compare the performance of classification models. 


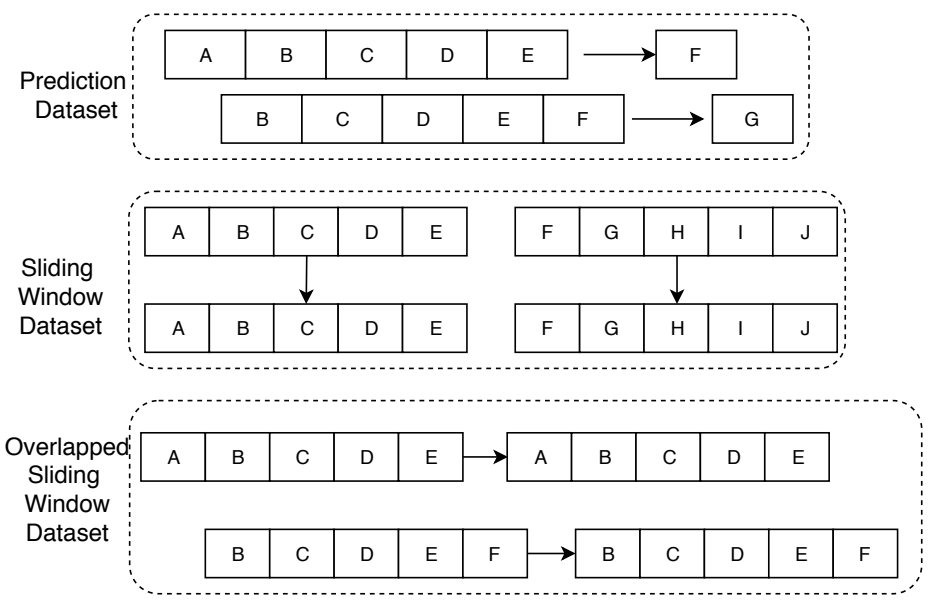

Figure 6.1: Feature preparation for prediction and pattern recognition methods. In prediction dataset, the observation window moves one step ahead and uses the next data point as target. In sliding window dataset and overlapped sliding window dataset, the observation window moves $L A G$ steps and one step ahead, respectively. In both cases, the data points in the observation windows are used as target [31].

\section{Experimental design}

The raw dataset used in the experiment are $\mathrm{CO}_{2}$ data collected from an elderly house in Norrköping, Sweden. According to data analysis, the day time and night time $\mathrm{CO}_{2}$ data roughly conform to two normal distributions with different means $(\mu)$ and standard deviations $(\sigma)$. In order to get a normal dataset, data cleaning is performed on the raw data. In this step, data points within $[\mu-3 \sigma, \mu+3 \sigma]$ are reserved while the rest are treated as abnormal values and repeatedly replaced with the average of neighbouring values until anomalous points are vanished. The cleaned dataset $D_{\text {normal }}$ is split into a normal training set $D_{\text {train }}$ and a normal test set $D_{\text {test }}$, which account for $80 \%$ and $20 \%$ data points, respectively.

After this step, two anomalous test sets $A_{\text {point }}$ and $A_{\text {context }}$ for point anomaly and contextual anomaly scenarios are generated as well as the corresponding gold standards. For point anomalies, values generated by a uniform function within $[$ minimal, $\mu-3 \sigma]$ and $[\mu+3 \sigma$, maximal $]$ are used to contaminate the normal values. For contextual anomalies, several consecutive data points are replaced with values that are 12 to 18 hours far from the originals to guarantee an obvious contextual difference. In both scenarios, a total $5 \%$ of the data points in the normal dataset are replaced with generated anomalous values. When the normal and anomalous datasets, i.e., $D_{\text {normal }}$, $D_{\text {test }}, A_{\text {point }}$ and $A_{\text {context }}$, are generated, real training and test sets used in 
Table 6.1: A comparison of point and contextual anomaly detection for $\mathrm{CO}_{2}$ using prediction (PD), sliding window (SWD) and overlapped sliding window dataset (OSWD). Autoencoder and LSTM-encoder-decoder top the others in point and contextual anomaly detection respectively, by comprehensively considering the network complexity, training time, area under curve (AUC), and optimal true positive rate (TPR) and false positive rate (FPR) metrics. (White cells: point anomaly detection results. Grey cells: contextual anomaly detection results.) [31]

\begin{tabular}{|c|c|c|c|c|c|c|c|c|c|}
\hline Model & $\begin{array}{l}\text { Hidden } \\
\text { layers }\end{array}$ & Neurons & $\begin{array}{l}\text { Total } \\
\text { parame- } \\
\text { ters }\end{array}$ & $\begin{array}{l}\text { Training } \\
\text { time (s) }\end{array}$ & $\begin{array}{l}\text { MAE } \\
\text { (D_test) }\end{array}$ & $\begin{array}{l}\text { MSE } \\
\text { (D_test) }\end{array}$ & AUC & $\begin{array}{l}\text { Opt. } \\
\text { TPR } \\
(\%)\end{array}$ & $\begin{array}{l}\text { Opt. } \\
\text { FPR } \\
(\%)\end{array}$ \\
\hline \multirow{2}{*}{ Regression (PD) } & \multirow{2}{*}{ / } & \multirow{2}{*}{ / } & \multirow{2}{*}{ / } & \multirow{2}{*}{1.8} & 4.7 & 42.2 & 0.9895 & 98.1 & 4.9 \\
\hline & & & & & 4.7 & 42.1 & 0.6606 & 61.9 & 40.2 \\
\hline \multirow{2}{*}{ ANN (PD) } & \multirow{2}{*}{3} & \multirow{2}{*}[200,200,50,1]{} & \multirow{2}{*}{52501} & \multirow{2}{*}{60} & 5.6 & 52.9 & 0.9932 & 97.6 & 4.0 \\
\hline & & & & & 4.7 & 42.0 & 0.6902 & 58.8 & 31.7 \\
\hline \multirow{2}{*}{ LSTM (PD) } & \multirow{2}{*}{1} & \multirow{2}{*}[128,1]{} & \multirow{2}{*}{66689} & \multirow{2}{*}{230} & 4.8 & 42.5 & 0.9891 & 98.6 & 5.1 \\
\hline & & & & & 4.8 & 42.6 & 0.6591 & 53.0 & 28.8 \\
\hline \multirow{2}{*}{ bi-LSTM (PD) } & \multirow{2}{*}{2} & \multirow{2}{*}[256,1]{} & \multirow{2}{*}{133377} & \multirow{2}{*}{380} & 4.7 & 41.9 & 0.9907 & 98.1 & 4.8 \\
\hline & & & & & 8.1 & 99.9 & 0.6066 & 52.9 & 37.8 \\
\hline \multirow{2}{*}{ ANN (TF) } & \multirow{2}{*}{4} & \multirow{2}{*}[200,200,50,1]{} & \multirow{2}{*}{54701} & \multirow{2}{*}{60} & 10.1 & 142.1 & 0.9947 & 97.6 & 3.5 \\
\hline & & & & & 8.0 & 99.1 & 0.6898 & 58.2 & 29.9 \\
\hline \multirow{2}{*}{ AE (SWD) } & \multirow{2}{*}{4} & & & 10 & 2.9 & 15.8 & 0.9969 & 98.6 & 0.9 \\
\hline & & {$[128,32,128,10]$} & 11050 & 10 & 3.3 & 30.3 & 0.8770 & 77.2 & 13.9 \\
\hline & & & & & 2.2 & 12.6 & 0.9981 & 98.5 & 1.8 \\
\hline LSIM (SWD) & 2 & {$[128,10]$} & 66689 & 30 & 3.0 & 48.1 & 0.8587 & 76.7 & 19.8 \\
\hline hi-l STM (SWD) & 2 & {$\left[\begin{array}{ll}256 & 10\end{array}\right]$} & 133377 & 40 & 2.9 & 16.5 & 0.9976 & 98.5 & 1.3 \\
\hline DI-LC TIVI (JVVD) & 2 & {$[250,10]$} & 15301 & 40 & 4.3 & 78.7 & 0.8648 & 80.2 & 16.1 \\
\hline LSTM multi- & 6 & {$[128,64,32$, } & 252161 & 90 & 11.2 & 411.4 & 0.9947 & 97.9 & 3.1 \\
\hline layer (SWD) & 6 & $64,128,10]$ & 252161 & 90 & 6.0 & 187.5 & 0.8692 & 78.7 & 19.1 \\
\hline & & & & & 4.4 & 33.3 & 0.9969 & 99.0 & 2.1 \\
\hline LSIM-ED (SWD) & 3 & {$[128,128,10]$} & 198273 & 50 & 6.0 & 189.6 & 0.8757 & 80.7 & 14.8 \\
\hline LSTM-ED multi- & 5 & {$[128,64,64$} & 247937 & 80 & 7.3 & 170.8 & 0.9873 & 98.2 & 5.6 \\
\hline layer (SWD) & 5 & $128,10]$ & & & 10.5 & 558.9 & 0.8711 & 76.2 & 10.3 \\
\hline AE (OSWD) & 4 & {$\left[\begin{array}{llll}128 & 32 & 128 & 10\end{array}\right]$} & 11050 & 40 & 0.2 & 0.08 & 0.9967 & 98.9 & 2.6 \\
\hline AE (USVVD) & 4 & {$[128,32,128,10]$} & 11050 & 40 & 0.14 & 0.04 & 0.7607 & 84.2 & 34.4 \\
\hline & & & & & 0.06 & 0.006 & 0.9691 & 92.9 & 4.4 \\
\hline LSIM (OSWD) & 2 & {$[128,10]$} & 66689 & 270 & 0.45 & 0.27 & 0.7792 & 81.6 & 30.6 \\
\hline LSTM-ED & 3 & {$\left[\begin{array}{lll}128 & 128 & 10\end{array}\right]$} & 198273 & 500 & 5.9 & 66.4 & 0.9911 & 98.1 & 5.9 \\
\hline (OSWD) & 3 & & & & 4.9 & 45.1 & 0.8635 & 83.9 & 32.2 \\
\hline SVM & 1 & 1 & 1 & 1 & 1 & 1 & 0.96 & 86.6 & 9.6 \\
\hline SVIVI & / & / & / & / & / & I & 0.48 & l & I \\
\hline Isolate forest & 1 & 1 & 1 & 1 & 1 & 1 & 0.9525 & 90.5 & 7.3 \\
\hline Isolate forest & / & / & / & / & / & / & 0.47 & l & I \\
\hline PCA & 1 & 1 & 1 & 1 & 1 & 1 & 0.6845 & 78.6 & 48.5 \\
\hline & 1 & 1 & 7 & 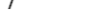 & 1 & 1 & 0.69 & 61.2 & 24.9 \\
\hline
\end{tabular}

the performance evaluation are prepared according to Figure 6.1, catering to the needs of prediction and pattern recognition models.

\section{Results}

Table 6.1 shows the evaluation results of point anomaly detection (white cells) and contextual anomaly detection (grey cells), which includes model parameters, training time, and performance metrics such as AUC.

For point anomaly detection, among all benchmarked models, the AE model achieves a high AUC value (0.9969) with surprisingly limited parameters and short training time, which greatly surpasses the other models considering the performance, network complexity and training difficulty. 
For contextual anomaly detection, in general, pattern recognition-based methods have higher AUC values than prediction-based methods, which shows pattern recognition is more efficient in identifying contextual anomalies. Overall, the performance of the LSTM-ED ensemble model with a sliding window dataset overtakes the others.

To validate the results, the same experiment is performed on a new dataset of temperature data collected in the university lab, which features a similar day and night fluctuation pattern and also a weekly pattern. The validation results are in accordance with that of the $\mathrm{CO}_{2}$ dataset, suggesting that the autoencoder model is optimal for detecting point anomalies while the LSTMED model is most suitable for contextual anomaly detection in an indoor environment.

The experimental details such as raw data distributions and plots, data cleaning, anomalous data generation, feature preparation, ROC graphs and the evaluation results for the validation dataset are presented in Paper V. 



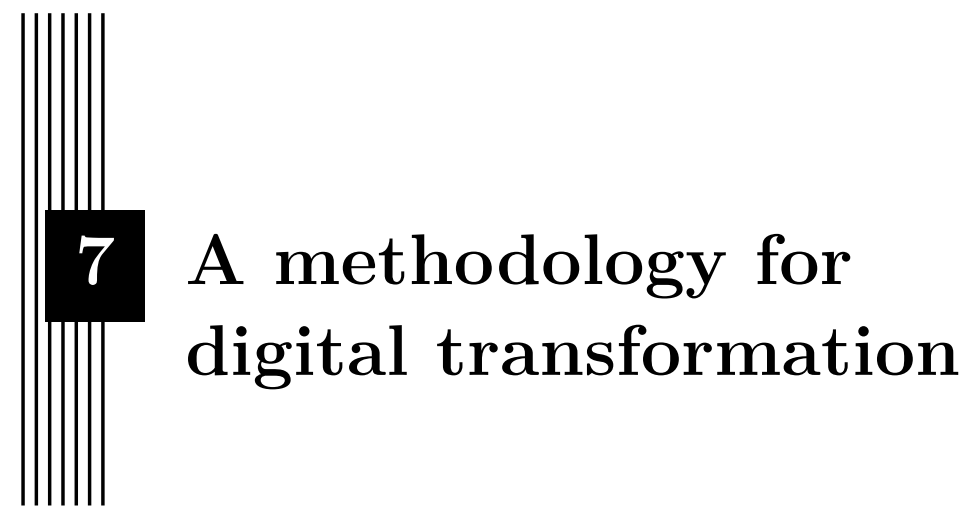

In this chapter, a reference framework is introduced as a guideline for small and medium sized enterprises (SMEs) within the manufacturing industry to utilize the Internet of things and cloud computing to accelerate their pace in the journey of digital transformation. Several design principles that are derived from real industrial practices are presented.

\subsection{Background}

Digital transformation has been taking place across all industries at different paces. The goal of digital transformation is to leverage newly emerging digital technologies such as IoT and cloud computing to enhance products and services, improve operation efficiency, automate industrial processes, optimize customer experiences, and transform business models. Many studies have investigated the strategies to perform digital transformation in the industry $[32,33,34]$ while others have looked into the challenges on the way towards digitalization $[35,36,37]$. In practice, digital transformation is easier to articulate than executing it [38]. This is especially a crucial issue for SMEs within the traditional manufacturing industry, which lack expertise in information and communication technologies (ICT) and are restricted by limited resource. However, a winning strategy to implement digital transformation can be more significant to SMEs to get first-mover advantage in the digital competition. Catering to the needs, an applicable framework is proposed to accelerate the digital transformation procedure in SMEs. The framework originates from a 
collaborative project between Linköping University and Vertical Plant Systems AB (VPS), which can be an guideline for industrial practitioners to design their digital roadmap and solutions.

\subsection{Digital transformation framework}

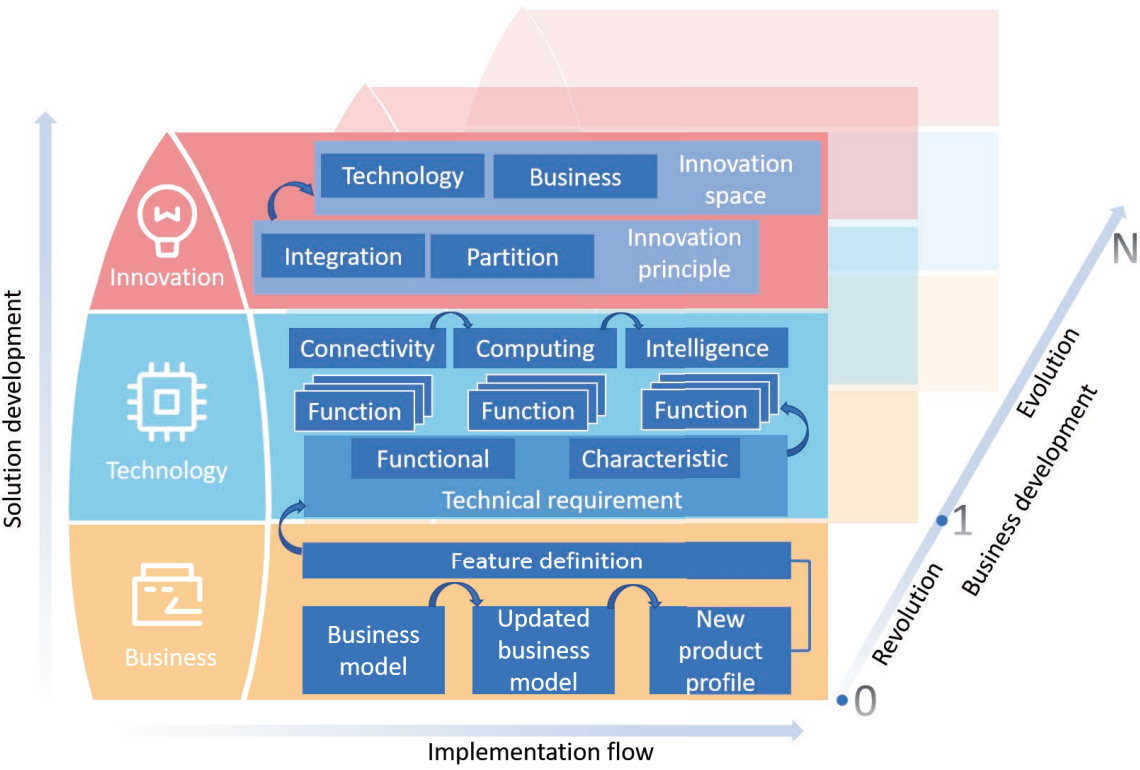

Figure 7.1: A reference framework for SMEs to accomplish digital transformation with enabling technologies, i.e., IoT and cloud computing. It features a three-stage iteration that covers consideration of business, technology and innovation, and three dimensions of development flows. The framework can be referenced by business stakeholders as a guideline to formulate technology development roadmap and to trigger business development [39]

Figure. 7.1 shows the proposed framework for digital transformation. It illustrates a three-stage rocket architecture to reflect the considerations of business, technology, and innovation aspects in a bottom-up order, which suggests digital transformation procedure should be business driven, technologyenabled, and innovation-guided. The details as explained as follows.

The framework starts from the business stage, which is in line with the fact that the origin of digital transformation is the business value. Therefore, a business model shall be defined or updated to reflect new value creations, customer segment and relation changes, and revenue streams. After that, the key features of the product can be extracted according to the result, which also forms the fundamentals for the next stage. 
The technology stage intends to provide a structured thinking to devise the technical solutions to achieve the anticipated goal. Based on the feature definition from the business stage, technical requirements that include both functional and characteristic requirements are formulated. Each feature is verified by corresponding requirements so as to generate a group of function blocks that are appropriately determined to address the requirements. In this step, enabling technologies in the ICT domain can be freely selected to fulfill the technical requirements. The final digital solution is derived from a union of all function blocks. Specifically, three progressive phases namely connectivity, computing, and intelligence are proposed as a guideline to implement the solution.

In the top stage, two innovation principles, namely partition and integration are proposed, which can be applied to either technology or business or both domains. Technical innovation can bring new business values while an innovation in business model can also trigger off new demand on technology. In either situation, the three stage procedure shall be revisited and iterated.

In addition to the three stages, three dimensions of development flows are proposed in the framework. The implementation and solution development flows guide the direction of thinking within and across the three stages, respectively. The dimension of business development lies in iterations of the three-stage procedure, within which the first iteration is seen as a revolution phase that bridges the gap from "0" to " 1 ". The following iterations constitute the evolution phase that pushes "1" towards "N". Following the three stage procedure and the three dimensions of development flows, digital transformation can be accomplished in a step-by-step manner. A case study of leveraging the proposed framework to perform digital transformation in the vertical plant wall industry is detailed in Paper VI.

\subsection{Design principles}

During the development of IoT solutions for SMEs, several design principles that are concluded from the VPS project can also be considered.

Time-to-market first, and prototyping fast. This principle originates from the agile development method that has been pervasively adopted in the software development process, and is equivalently significant for SMEs to develop IoT solutions. It implies that SMEs should not expect a fully polished solution to be achieved before it is delivered to customers but are encouraged to prototype fast and deploy in an early stage. The product and service shall be continuously improved through on-site tests and feedback from users. This strategy guarantees the pitfalls in the prototype can be identified at an early time and the solution is verified in a long period. 
Cloud first. Cloud computing is the most important enabler to the landing of IoT technologies, benefiting from its high reliability, availability, scalability and relative low cost compared to implementing private solutions. By taking advantage of cloud computing, SMEs can greatly accelerate the development pace and ease the efforts put into digital transformation. Our previous research result has led to the Culturebee system (http://culturebee.se), which is a wireless monitoring and control system for Swedish churches and is based on self-maintained private cloud. However, the solution can hardly be accepted by SMEs like VPS as the cost is a burden. Therefore, in the plant wall project, a public cloud-based solution is proposed. Toolsets like IoT Hub and IoT software development kit (SDK) offered by Microsoft Azure Cloud can effectively handle trivial functions such as network connection and device management so that we can focus on the implementation of core functions regarding plant walls. With limited monthly cost, compute and storage services in the cloud can be flexibly subscribed and scaled according to needs while eliminating the necessity to maintain hardware infrastructures.

Evolving with new technologies. Technologies in IoT and cloud computing are rapidly advancing. It is a good practice to keep an eye on emerging technologies and drive the evolution of digital solution with new technologies. For instance, as the cloud computing architecture is evolving towards an edge-cloud computing architecture, and the container technology-based cloud native programming pattern becomes a novel trend in the industry, in the plant wall project, the edge-cloud architecture is benchmarked and put into practice. Based on the new architecture, a part of the cloud functions is offloaded to the edge computing infrastructure that is deployed to the local field to enable capabilities such as data pre-processing and offline storage. Applications are also developed and deployed as isolated container modules to guarantee continuous and large-scale deployment.

Asset-light first. During the development, SMEs should carefully balance the resources spent on the realization of each function block and focus on implementation of functions tightly related to core value creation. In the practice of VPS, major resources are spent on the implementation of a digital platform that can offer remote sensing and maintenance capabilities, and big data-enabled smart functions like anomaly detection and alarm. These asset-light functions will consolidate the business value of VPS after the digital transformation thus are prioritized. Other tasks such as manufacturing, selection of sensors, and hardware component development are either handled to partners or ordered from market, which maximizes the resources invested to the right direction. 


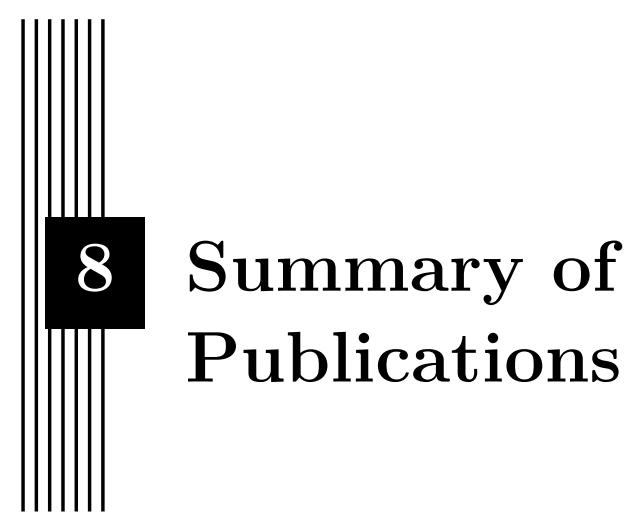

This chapter summaries the papers that are associated with this thesis.

Paper I: Y. Liu, K. Akram Hassan, M. Karlsson, Z. Pang and S. Gong, "A Data-Centric Internet of Things Framework Based on Azure Cloud," in IEEE Access, vol. 7, pp. 53839-53858, 2019.

Summary: In this study, a data-centric IoT framework is proposed, which incorporates three promising protocols with fundamental security schemes, i.e., WiFi, Thread and LoRaWAN, to cater to massive IoT and broadband IoT use cases in local, personal, and wide area networks. By taking advantages of the Azure cloud infrastructure, the framework features a unified device management model and data model to conquer the interoperability, co-existence, device management and data consistency challenges in IoT practices. The system architecture is provided and the implementation details are described as a reference to Io $\mathrm{T}$ practitioners.

Contribution: Proposed a data-centric IoT framework exemplified with three most representative protocols with inherent high level security property, eased the interoperability, co-existence, device management and data consistency challenges in IoT practice, validated the framework with a reference implementation and a practical case study. 
Paper II: Y. Liu, Z. Pang, G. Dán, D. Lan and S. Gong, "A Taxonomy for the Security Assessment of IP-Based Building Automation Systems: The Case of Thread," in IEEE Transactions on Industrial Informatics, vol. 14, no. 9, pp. 4113-4123, Sept. 2018.

Summary: In this study, a security assessment taxonomy for building automation networks is proposed with consideration of the whole life cycle of a network device. The taxonomy is then applied to the security analysis of Thread protocol. The results show that Thread has superior security performance than its competitors. Several security enhancement measures are also proposed to strengthen the security level of Thread.

Contribution: Proposed the security assessment taxonomy, performed the security analysis of the Thread protocol and proposed countermeasures to enhance Thread security.

Paper III: Y. Liu, D. Lan, Z. Pang, M. Karlsson and S. Gong, "Performance evaluation of containerization in edge-cloud computing stacks for industrial applications: a client perspective," in IEEE Open Journal of the Industrial Electronics Society, vol. 2, pp. 153-168, 2021, doi: 10.1109/OJIES.2021.3055901.

Summary: In this study, from the industry client perspective, a performance evaluation methodology is proposed and applied to the state-of-the-art containerization-based edge-cloud computing infrastructure. The influence of message sending interval, payload, network bandwidth, and number of concurrent devices on full-stack latency are evaluated. The processing capabilities of executing machine learning tasks are measured. The results show that containerization on the edge does not introduce noticeable performance decrease in communication, computing and intelligence capabilities, which can be a promising direction for the edge-cloud computing paradigm. However, current edge-cloud infrastructure shall be largely improved to fulfill the performance requirements posed by critical industrial applications. It is also showcased that partitioning of industrial applications into microservices throughout the full stack can be considered at the beginning of solution design.

Contribution: Performed a full stack analysis of latency performance for the containerization technology used in the state-of-the-art edge-cloud computing infrastructure, evaluated the processing capabilities of containerization in terms of executing machine learning and neural network tasks in edge-cloud computing stacks, profiled the performance limits and highlighted the necessity of partitioning in existing edge-cloud infrastructure in industrial practice. 
Paper IV: Y. Liu, K. Akram Hassan, M. Karlsson, O. Weister and S. Gong, "Active Plant Wall for Green Indoor Climate Based on Cloud and Internet of Things," in IEEE Access, vol. 6, pp. 33631-33644, 2018.

Summary: In this study, a remote monitoring and control system that is specific to plant walls is proposed. The system utilizes the Internet of Things technology and the Azure public cloud platform to automate the management procedure, improve the scalability, enhance user experiences of plant walls, and contribute to a green indoor climate. Compared to other solutions, our solution features in bi-directional communications, native-IP support, easy deployment, reliability and scalability, and low cost.

Contribution: Proposed a complete cloud-enabled solution for remote monitoring and management of plant wall systems.

Paper V: Y. Liu, M. Karlsson, Z. Pang and S. Gong, "Anomaly detection based on machine learning in IoT-based vertical plant wall for indoor climate control," in Building and Environment, vol. 183, 2020.

Summary: In this paper, the possibility of applying machine learning based anomaly detection methods to vertical plant wall systems is investigated, which aims to enhance the automation and improve the intelligence so as to realize predictive maintenance of the indoor climate. Two categories of anomalies, namely point anomalies and contextual anomalies are researched. Prediction-based and pattern recognition-based methods are investigated and applied to indoor climate anomaly detection. The results show that neural network-based models, specifically the autoencoder (AE) and the long shortterm memory encoder decoder (LSTM-ED) model surpass the others in terms of detecting point anomalies and contextual anomalies, respectively, therefore can be deployed into vertical plant walls systems in industrial practice.

Contribution: Investigated the performance of both prediction and pattern recognition-based anomaly detection methods on both point and contextual anomalies in a real indoor $\mathrm{CO}_{2}$ concentration dataset and validate the results with a real temperature dataset, proposed a prediction-based method for data preprocessing to eliminate point anomalies and implemented realtime anomaly detection functions in the Azure cloud platform as a proof of concept, showcased an exploitation of novel machine learning and Internet of Things technologies into building environment science to speed up the solution development.

Paper VI: Y. Liu, Z. Ni, M. Karlsson, Z. Pang and S. Gong, "Methodology for digital transformation with Internet of things and cloud computing: 
a practical guideline for innovation to small and medium-sized enterprises", Manuscript, 2021.

Summary: In this paper, a reference framework for SMEs is proposed as a guideline towards digital transformation. The framework features a threestage procedure that covers business, technology and innovation, which can be iterated to drive the product and business development. A case study about digital transformation taking place in the vertical plant wall industry is presented. Furthermore, some solution design principles that are concluded from industrial practice are discussed. This paper reviews earlier publications of the authors that are related to digital transformation practice and aims to accelerate the pace of SMEs in the journey of digital transformation.

Contribution:Proposed an applicable framework for SMEs in traditional manufacturing industry to drive IoT and cloud computing enabled digital transformation, exemplified the framework with a real case study in the vertical plant wall industry, provided instructive principles for solution design to accelerate the digitalization process and reduce risk of failure. 


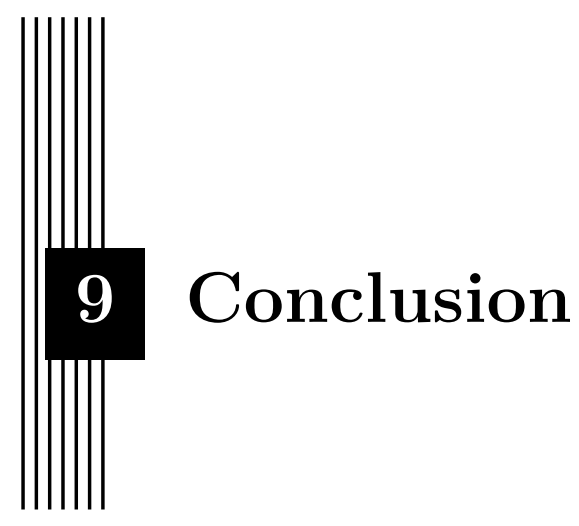

Since the emergence of the concept, IoT has been a fashion term appreciated by the research and industrial society. Together with the progress in information and communication technologies such as wireless sensor networks and system on chips, IoT is evolving towards a fully-fledged paradigm and is anticipated to reshape human daily life and industrial production with large scale deployment of sensors, massive volume of data collection, and intelligence derived from accumulated data. In the past, IoT innovations were mainly isolated within individual applications or subjects while the popularity of IoT has experienced fluctuations. The emergence of cloud computing has become an unprecedented power to accelerate IoT development. Benefiting from the superior computation and connectivity capability, unlimited and flexible storage, scalable service subscription and ever-growing intelligence, IoT deployment can be largely simplified by taking advantage of the cloud infrastructure. However, a series of challenges are identified and cannot be resolved by the cloud suppliers as their efforts are dedicated to software and platform services but can hardly contribute to the end devices due to the heterogeneity in hardware and communication protocols.

This dissertation aims to provide a holistic approach to enable the landing of IoT. A data-centric IoT framework based on public cloud is proposed to tackle the encountered challenges, i.e., the interoperability and compatibility among link-layer protocols, the uniform device management scheme, and the heterogeneity of massive data from diverse IoT applications. The framework leverages WiFi, Thread, and LoRaWAN network protocols to cover personal 
area, local area, and wide area network use cases, and features a uniform device management model and data communication model. A security analysis taxonomy is proposed as a tool to enhance security assessment in IoT field networks in the framework. Furthermore, catering to the industry tendency that cloud computing is gradually migrating towards the edge-cloud computing paradigm, edge computing is considered as a complement to the IoT framework, in which the cloud native programming paradigm is highlighted, and container-based virtualization is employed. The performance of the edgecloud computing stack in terms of communication, computing and intelligence is comprehensively evaluated and the results show that containerization-based edge-cloud computing architecture represents a promising future in IoT applications. A real industry application, i.e., the vertical plant wall system is used as a case study. Based on the IoT framework, a remote monitoring and management system for vertical plant walls is developed. It is also showcased that novel machine learning and neural networks models can be utilized to achieve the ultimate goal of IoT - the intelligence. A practical example, i.e., anomaly detection for indoor climate based on collected environmental data from the vertical plant wall, is presented. Lastly, in light of the fact that SMEs are encountering difficulties in IoT-enabled digital transformation, a reference framework for SMEs is proposed as an easy-to-follow guideline to accelerate the digital transformation to take place.

The proposed IoT framework is specific for applications within the massive IoT and broadband IoT categories. The research results also identify that existing edge-cloud infrastructure can hardly meet the quantitative performance requirements for critical industrial IoT applications. Therefore, in the future, an edge-cloud infrastructure that caters to the demanding requirements posed by industrial IoT shall be investigated. Besides, edge devices are to be widespread deployed in the upcoming $5 \mathrm{G}$ era, therefore edge intelligence in the edge-cloud computing paradigm which is a vital topic to realize the true value of IoT shall be further studied. 


\section{Bibliography}

[1] Luigi Atzori, Antonio Iera, and Giacomo Morabito. "The Internet of Things: A survey." In: Computer Networks 54.15 (2010), pp. 2787-2805. ISSN: 1389-1286. DOI: https://doi.org/10.1016/j.comnet.2010.05. 010. URL: https://www.sciencedirect.com/science/article/pii/ S1389128610001568.

[2] Jan Höller, Vlasios Tsiatsis, Catherine Mulligan, Stamatis Karnouskos, Stefan Avesand, and David Boyle. "Chapter 2 - M2M to IoT - The Vision." In: From Machine-To-Machine to the Internet of Things. Ed. by Jan Höller, Vlasios Tsiatsis, Catherine Mulligan, Stamatis Karnouskos, Stefan Avesand, and David Boyle. Oxford: Academic Press, 2014, pp. 937. ISBN: 978-0-12-407684-6. DOI: https ://doi .org/10.1016/B978-012-407684-6.00002-4.

[3] Yu Liu. A Data-centric Internet of Things Framework Based on Public Cloud. 2019. DOI: 10.3384/lic.diva-159770.

[4] Cellular IoT Evolution for Industry Digitalization. White Paper. Ericsson, Jan. 2019.

[5] A. Zanella, N. Bui, A. Castellani, L. Vangelista, and M. Zorzi. "Internet of Things for Smart Cities." In: IEEE Internet of Things Journal 1.1 (Feb. 2014), pp. 22-32. ISSN: 2327-4662. DOI: 10 . 1109 / JIOT . 2014. 2306328.

[6] Massive IoT in the City. Report. Ericsson, Nov. 2016. 
[7] S. Gangakhedkar, H. Cao, A. R. Ali, K. Ganesan, M. Gharba, and J. Eichinger. "Use Cases, Requirements and Challenges of 5G Communication for Industrial Automation." In: 2018 IEEE International Conference on Communications Workshops (ICC Workshops). 2018, pp. 16.

[8] Filip Holik. "Meeting Smart City Latency Demands with SDN." In: Intelligent Information and Database Systems: Recent Developments. Ed. by Maciej Huk, Marcin Maleszka, and Edward Szczerbicki. Cham: Springer International Publishing, 2020, pp. 43-54. ISBN: 978-3-03014132-5. DOI: 10 . 1007/978-3-030-14132-5_4. URL: https : //doi . org/10.1007/978-3-030-14132-5_4.

[9] J. Hiller, M. Henze, M. Serror, E. Wagner, J. N. Richter, and K. Wehrle. "Secure Low Latency Communication for Constrained Industrial IoT Scenarios." In: 2018 IEEE 43rd Conference on Local Computer Networks (LCN). 2018, pp. 614-622. DOI: 10.1109/LCN.2018.8638027.

[10] Y. Liu, D. Lan, Z. Pang, M. Karlsson, and S. Gong. "Performance Evaluation of Containerization in Edge-Cloud Computing Stacks for Industrial Applications: A Client Perspective." In: IEEE Open Journal of the Industrial Electronics Society 2 (2021), pp. 153-168. DOI: 10.1109/0JIES. 2021.3055901.

[11] Carlos Bravo and Henrik Bäckström. Edge computing and deployment strategies for communication service providers. White Paper. Ericsson, 2020.

[12] Y. Liu, K. Akram Hassan, M. Karlsson, Z. Pang, and S. Gong. "A Data-Centric Internet of Things Framework Based on Azure Cloud." In: IEEE Access 7 (2019), pp. 53839-53858. ISSN: 2169-3536. DOI: 10.1109/ ACCESS . 2019. 2913224.

[13] Microsoft. Microsoft Azure IoT reference architecture. Sept. 2018. URL: https : / / azure . microsoft . com / en-us / blog / azure-iotreference-architecture-2-1-release/.

[14] Y. Liu, Z. Pang, G. Dán, D. Lan, and S. Gong. "A Taxonomy for the Security Assessment of IP-Based Building Automation Systems: The Case of Thread." In: IEEE Transactions on Industrial Informatics 14.9 (Sept. 2018), pp. 4113-4123. ISSN: 1551-3203. DOI: 10.1109/TII. 2018. 2844955.

[15] D. J. Fehér and B. Sandor. "Effects of the WPA2 KRACK Attack in Real Environment." In: 2018 IEEE 16th International Symposium on Intelligent Systems and Informatics (SISY). Sept. 2018, pp. 000239000242.

[16] WiFi Alliance. Next generation Wi-Fi: The future of connectivity. Dec. 2018. URL: https://www.wi-fi.org. 
[17] LoRaWAN Security. White paper. LoRa Alliance, Feb. 2017.

[18] Microsoft. Azure IoT Hub Guide. 2019. URL: https : / / docs . microsoft.com/en-us/azure/iot-hub.

[19] Kubernetes. URL: https://kubernetes.io/.

[20] Azure IoT Edge. URL: https://docs.microsoft.com/en-us/azure/ iot-edge/.

[21] Amazon Greengrass. URL: https://aws.amazon.com/greengrass/.

[22] IBM Edge Application Manager. URL: https://developer.ibm.com/ components/ibm-edge-application-manager/.

[23] KubeEdge. URL: https://kubeedge.io/en/.

[24] Jeffrey S. Katz. AIoT: Thoughts on Artificial Intelligence and the Internet of Things. 2019. URL: https : //iot.ieee.org/conferencesevents/wf-iot-2014-videos/56-newsletter/july-2019.html.

[25] Andrejs Bondarevs, Patrik Huss, Shaofang Gong, Ola Weister, and Roger Liljedahl. "Green Walls Utilizing Internet of Things." In: Sensors and Transducers 192 (2015), pp. 16-21. ISSN: 2306-8515.

[26] Vertical Plant Systems AB. URL: https : / / www . verticalplantssystem.com.

[27] Y. Liu, K. Akram Hassan, M. Karlsson, O. Weister, and S. Gong. "Active Plant Wall for Green Indoor Climate Based on Cloud and Internet of Things." In: IEEE Access 6 (2018), pp. 33631-33644. ISSN: 2169-3536. DOI: $10.1109 /$ ACCESS . 2018.2847440.

[28] Xilei Dai, Junjie Liu, and Xin Zhang. "A review of studies applying machine learning models to predict occupancy and window-opening behaviours in smart buildings." In: Energy and Buildings 223 (2020), p. 110159. ISSN: 0378-7788. DOI: https : // doi .org/10.1016/j . enbuild . 2020 . 110159. URL: http : / / www . sciencedirect . com / science/article/pii/S0378778820303017.

[29] Karl Mason and Santiago Grijalva. "A review of reinforcement learning for autonomous building energy management." In: Computers \& Electrical Engineering 78 (2019), pp. 300-312. ISSN: 0045-7906. DOI: https:// doi .org/10.1016/j . compeleceng . 2019.07.019. URL: http: //www . sciencedirect.com/science/article/pii/S0045790618333421.

[30] Mohammed Ayoub. "A review on machine learning algorithms to predict daylighting inside buildings." In: Solar Energy 202 (2020), pp. 249-275. ISSN: 0038-092X. DOI: https://doi .org/10 . 1016/j . solener . 2020 . 03. 104. URL: http : //www . sciencedirect.com/science/article/ pii/S0038092X20303509. 
[31] Y. Liu, Z. Pang, M. Karlsson, and S. Gong. "Anomaly detection based on machine learning in IoT-based vertical plant wall for indoor climate control." In: Building and Environment 183 (2020). ISSN: 0360-1323. URL: https : //doi .org/10.1016/j.buildenv . 2020.107212 . .

[32] A. Zimmermann, R. Schmidt, K. Sandkuhl, M. Wißotzki, D. Jugel, and M. Möhring. "Digital Enterprise Architecture - Transformation for the Internet of Things." In: 2015 IEEE 19th International Enterprise Distributed Object Computing Workshop. 2015, pp. 130-138. DOI: 10.1109/ EDOCW. 2015.16.

[33] A. A. Pflaum and P. Gölzer. "The IoT and Digital Transformation: Toward the Data-Driven Enterprise." In: IEEE Pervasive Computing 17.1 (2018), pp. 87-91. DOI: 10.1109/MPRV.2018.011591066.

[34] A. Zimmermann, R. Schmidt, K. Sandkuhl, D. Jugel, J. Bogner, and M. Möhring. "Evolution of Enterprise Architecture for Digital Transformation." In: 2018 IEEE 22nd International Enterprise Distributed Object Computing Workshop (EDOCW). 2018, pp. 87-96. DOI: 10.1109/ EDOCW. 2018.00023.

[35] Marcus Wolf, Arlett Semm, and Christian Erfurth. "Digital Transformation in Companies - Challenges and Success Factors." In: Innovations for Community Services. Ed. by Michal Hodoň, Gerald Eichler, Christian Erfurth, and Günter Fahrnberger. Cham: Springer International Publishing, 2018, pp. 178-193.

[36] Thomas Hess, Christian Matt, Alexander Benlian, and Florian Wiesböck. "Options for Formulating a Digital Transformation Strategy." In: MIS Quarterly Executive 15 (June 2016), pp. 123-139.

[37] A. Aagaard, M. Presser, and T. Andersen. "Applying Iot as a leverage for business model innovation and digital transformation." In: 2019 Global IoT Summit (GIoTS). 2019, pp. 1-5. DOI: 10.1109/GIOTS. 2019. 8766397.

[38] Ina Sebastian, Jeanne Ross, Cynthia Beath, Martin Mocker, Kate Moloney, and Nils Fonstad. "How big old companies navigate digital transformation." In: MIS quarterly executive 16.3 (2017), pp. 197-213. URL: http://misqe.org/ojs2/index.php/misqe/article/view/783.

[39] Y. Liu, Z. Ni, M. Karlsson, Z. Pang, and S. Gong. "Methodology for digital transformation with Internet of things and cloud computing: a practical guideline for innovation to small and medium-sized enterprises." In: Manuscript (2021). 


\section{Papers}

The papers associated with this thesis have been removed for copyright reasons. For more details about these see:

http://urn.kb.se/resolve?urn=urn:nbn:se:liu:diva-175383 


\section{FACULTY OF SCIENCE AND ENGINEERING}

Linköping Studies in Science and Technology, Dissertation No. 2139, 2021 Department of Science and Technology

Linköping University

SE-581 83 Linköping, Sweden

www.liu.se 